\title{
THREE GRAVITATIONALLY LENSED SUPERNOVAE BEHIND CLASH GALAXY CLUSTERS
}

\author{
Brandon Patel ${ }^{1}$, Curtis McCully ${ }^{1}$, Saurabh W. Jha ${ }^{1}$, Steven A. Rodney ${ }^{2,34}$, David O. Jones ${ }^{2}$, Or Graur ${ }^{2,3,4,5}$, \\ Julian Merten $^{6}$, Adi Zitrin ${ }^{7,34}$, Adam G. Riess ${ }^{2,8}$, Thomas Matheson ${ }^{9}$, Masao Sako $^{10}$, Thomas W.-S. Holoien ${ }^{1}$, \\ Marc Postman $^{8}$, Dan CoE $^{8}$, Matthias Bartelmann ${ }^{11}$, Italo Balestra ${ }^{12,13}$, Narciso Benítez ${ }^{14}$, Rychard Bouwens ${ }^{15}$, \\ Larry Bradley $^{8}$, Tom Broadhurst ${ }^{16}$, S. Bradley Cenko ${ }^{17}, 18$, Megan Donahue $^{19}$, AleXei V. Filippenko ${ }^{18}$, \\ Holland Ford ${ }^{2}$, Peter Garnavich ${ }^{20}$, Claudio Grillo ${ }^{21}$, Leopoldo Infante $^{22}$, Stéphanie Jouvel ${ }^{23}$, Daniel Kelson ${ }^{24}$, \\ Anton Koekemoer ${ }^{8}$, Ofer Lahav $^{25}$, Doron Lemze ${ }^{2}$, Dan MaOz $^{3}$, Elinor Medezinski ${ }^{2}$, Peter Melchior $^{26}$, \\ Massimo Meneghetti ${ }^{6,27,28}$, Alberto Molino ${ }^{14}$, John Moustakas ${ }^{29}$, Leonidas A. MoustaKas ${ }^{6}$, Mario Nonino $^{12}$, \\ Piero Rosati $^{30,31}$, Stella Seitz ${ }^{32}$, Louis G. Strolger ${ }^{8}$, Keitchi Umetsu ${ }^{33}$, and Wei Zheng ${ }^{2}$ \\ ${ }^{1}$ Department of Physics and Astronomy, Rutgers, The State University of New Jersey, Piscataway, NJ 08854, USA; bpatel02@ physics.rutgers.edu \\ ${ }^{2}$ Department of Physics and Astronomy, The Johns Hopkins University, Baltimore, MD 21218, USA \\ ${ }^{3}$ School of Physics and Astronomy, Tel Aviv University, Tel Aviv 69978, Israel \\ ${ }^{4}$ Department of Astrophysics, American Museum of Natural History, New York, NY 10024, USA \\ 54 CCPP, New York University, 4 Washington Place, New York, NY 10003, USA \\ ${ }^{6}$ Jet Propulsion Laboratory, California Institute of Technology, MS 169-327, Pasadena, CA 91109, USA \\ ${ }^{7}$ Cahill Center for Astronomy and Astrophysics, California Institute of Technology, MS 249-17, Pasadena, CA 91125, USA \\ ${ }^{8}$ Space Telescope Science Institute, 3700 San Martin Drive, Baltimore, MD 21208, USA \\ ${ }^{9}$ National Optical Astronomy Observatory, 950 North Cherry Avenue, Tucson, AZ 85719, USA \\ ${ }^{10}$ Department of Physics and Astronomy, University of Pennsylvania, Philadelphia, PA 19104, USA \\ ${ }^{11}$ Institut für Theoretische Astrophysik, Universität Heidelberg, Zentrum für Astronomie, Philosophenweg 12, D-69120 Heidelberg, Germany \\ ${ }^{12}$ INAF-Osservatorio Astronomico di Trieste, Via G. B. Tiepolo 11, I-34131 Trieste, Italy \\ ${ }^{13}$ INAF-Osservatorio Astronomico di Capodimonte, via Moiariello 16, I-80131 Napoli, Italy \\ ${ }^{14}$ Instituto de Astrofísica de Andalucía (CSIC), Camino Bajo de Huétor 24, E-18008 Granada, Spain \\ ${ }^{15}$ Leiden Observatory, Leiden University, NL-2300 RA Leiden, The Netherlands \\ ${ }^{16}$ Department of Theoretical Physics, University of the Basque Country, P.O. Box 644, E-48080 Bilbao, Spain \\ ${ }^{17}$ Astrophysics Science Division, NASA/GSFC, Mail Code 661, Greenbelt, MD 20771, USA \\ ${ }^{18}$ Department of Astronomy, University of California, Berkeley, CA 94720-3411, USA \\ ${ }^{19}$ Department of Physics and Astronomy, Michigan State University, East Lansing, MI 48824, USA \\ ${ }^{20}$ Department of Physics, University of Notre Dame, Notre Dame, IN 46556, USA \\ ${ }^{21}$ Dark Cosmology Centre, Niels Bohr Institute, University of Copenhagen, Juliane Mariesvej 30, DK-2100 Copenhagen, Denmark \\ ${ }^{22}$ Institute of Astrophysics, Pontificia Universidad Católica de Chile, Av. Vic. Mackenna 4860, Santiago, Chile \\ ${ }^{23}$ Institut de Ciéncies de l'Espai (IEEC-CSIC), E-08193 Bellaterra (Barcelona), Spain \\ ${ }^{24}$ Observatories of the Carnegie Institution of Washington, Pasadena, CA 91101, USA \\ 25 Department of Physics and Astronomy, University College London, Gower Street, London WC1E 6BT, UK \\ ${ }^{26}$ Center for Cosmology and Astro-Particle Physics, and Department of Physics, The Ohio State University, \\ 191 West Woodruff Avenue, Columbus, OH 43210, USA \\ ${ }^{27}$ INAF-Osservatorio Astronomico di Bologna, and INFN, Sezione di Bologna, Via Ranzani 1, I-40127 Bologna, Italy \\ ${ }_{28}$ INFN, Sezione di Bologna, Viale Berti-Pichat 6/2, 40127, Bologana, Italy \\ ${ }^{29}$ Department of Physics and Astronomy, Siena College, 515 Loudon Road, Loudonville, NY 12211, USA \\ ${ }^{30}$ Dipartimento di Fisica e Scienze della Terra, Università di Ferrara, Via Saragat 1, I-44122 Ferrara, Italy \\ ${ }^{31}$ ESO-European Southern Observatory, D-85748 Garching bei München, Germany \\ 32 Universitäts-Sternwarte, München, Scheinerstr. 1, D-81679 München, Germany \\ ${ }^{33}$ Institute of Astronomy and Astrophysics, Academia Sinica, P.O. Box 23-141, Taipei 10617, Taiwan \\ Received 2013 December 3; accepted 2014 March 7; published 2014 April 9
}

\begin{abstract}
We report observations of three gravitationally lensed supernovae ( $\mathrm{SNe}$ ) in the Cluster Lensing And Supernova survey with Hubble (CLASH) Multi-Cycle Treasury program. These objects, SN CLO12Car $(z=1.28)$, SN CLN12Did $(z=0.85)$, and SN CLA11Tib $(z=1.14)$, are located behind three different clusters, MACSJ1720.2+3536 $(z=0.391)$, RXJ1532.9+3021 $(z=0.345)$, and A383 $(z=0.187)$, respectively. Each SN was detected in Hubble Space Telescope optical and infrared images. Based on photometric classification, we find that SNe CLO12Car and CLN12Did are likely to be Type Ia supernovae (SNe Ia), while the classification of SN CLA11Tib is inconclusive. Using multi-color light-curve fits to determine a standardized SN Ia luminosity distance, we infer that SN CLO12Car was $\sim 1.0 \pm 0.2$ mag brighter than field SNe Ia at a similar redshift and ascribe this to gravitational lens magnification. Similarly, SN CLN12Did is $\sim 0.2 \pm 0.2$ mag brighter than field SNe Ia. We derive independent estimates of the predicted magnification from CLASH strong+weak-lensing maps of the clusters (in magnitude units, $2.5 \log _{10} \mu$ ): $0.83 \pm 0.16 \mathrm{mag}$ for SN CLO12Car, $0.28 \pm 0.08 \mathrm{mag}$ for SN CLN12Did, and $0.43 \pm 0.11 \mathrm{mag}$ for SN CLA11Tib. The two SNe Ia provide a new test of the cluster lens model predictions: we find that the magnifications based on the SN Ia brightness and those predicted by the lens maps are consistent. Our results herald the promise of future observations of samples of cluster-lensed SNe Ia (from the ground or space) to help illuminate the dark-matter distribution in clusters of galaxies, through the direct determination of absolute magnifications.
\end{abstract}

Key words: cosmology: observations - galaxies: clusters: general - gravitational lensing: weak supernovae: general

Online-only material: color figures 


\section{INTRODUCTION}

Supernovae (SNe) have proven to be important tools for studying the universe. Type Ia $\mathrm{SNe}$ (SNe Ia) have been instrumental in the discovery that the expansion of the universe is accelerating, and probing the dark energy driving the acceleration (e.g., Riess et al. 1998; Perlmutter et al. 1999; Wood-Vasey et al. 2007; Kessler et al. 2009a; Hicken et al. 2009; Sullivan et al. 2011; Suzuki et al. 2012 and references therein). Corecollapse (CC) SNe trace the star-formation history of the universe (e.g., Dahlen et al. 2004; Bazin et al. 2009; Botticella et al. 2012). Gravitational lensing of SNe can augment their cosmological utility, in both the strong- and weak-lensing regimes. The seminal paper of Refsdal (1964), which considered measuring galaxy masses and the Hubble parameter from lensed image time delays, in fact envisaged $\mathrm{SNe}$ as the background sources. ${ }^{35}$ While no multiply imaged SN has yet been discovered, several applications of lensed SNe are currently being explored.

Gravitational lensing allows massive galaxy clusters to be used as cosmic telescopes, increasing the apparent brightness of distant sources that would otherwise be too faint to detect (Sullivan et al. 2000; Kneib et al. 2004; Stanishev et al. 2009). Ground-based near-infrared (IR) surveys of galaxy clusters are being used to find lensed SNe, including a CC SN with redshift $z=1.7$ (Goobar et al. 2009; Amanullah et al. 2011 and references therein). Lensed SNe Ia are particularly useful, as their standardizable luminosities allow for a direct measurement of the absolute lensing magnification (Kolatt \& Bartelmann 1998).

The Cluster Lensing And Supernova survey with Hubble (CLASH; Postman et al. 2012) has observed 25 galaxy clusters in 16 Hubble Space Telescope (HST) broadband filters covering the near-ultraviolet (UV) to the near-IR with the Advanced Camera for Surveys (ACS) and the Wide Field Camera 3 (WFC3). The WFC3 infrared channel (WFC3/IR) is especially important for finding and studying high-redshift SNe, efficiently surveying large sky areas for SNe whose peak flux has redshifted from the optical to the IR. The SN discoveries and follow-up observations from CLASH are discussed at length by Graur et al. (2014). Here we focus on SNe behind CLASH clusters (in the "prime" fields centered on the clusters); these have not been included in the Graur et al. (2014) "parallel" field sample.

All distant $\mathrm{SNe}$ are lensed (magnified, or more often, demagnified) to some extent by large-scale structure along the line of sight. This adds a statistical "nuisance" in cosmological inferences from $\mathrm{SNe}$ Ia that can be corrected either assuming an overall magnification distribution (e.g., Holz \& Linder 2005; Martel \& Premadi 2008) or with convergence estimates along each SN light of sight using simplified scaling relations applied to the nearby foreground galaxies observed (Kronborg et al. 2010; Jönsson et al. 2010; Smith et al. 2014, and references therein).

Individual $\mathrm{SNe}$ that are detectably magnified have been found only rarely in recent wide-field surveys. A gravitational lens has to be precisely aligned with the background source to produce large magnifications, and this is significantly rarer for $\mathrm{SNe}$

\footnotetext{
${ }^{34}$ Hubble Fellow.

35 Refsdal (1964) also presciently concluded with the idea that in addition to lensed SNe, "star-like objects with intense emission both in the radio range and optical range" that "have been recently discovered" may also be valuable lensed sources, "giving a possibility of testing the different (cosmological) models."
}

compared to background galaxies as sources. Their short-lived durations also make lensed SNe rarer than even lensed quasars (Oguri \& Marshall 2010). Moreover, the survey footprints of the Supernova Legacy Survey (SNLS) and the SDSS-II Supernova Survey did not contain many massive galaxy clusters to act as lenses for background SNe (Graham et al. 2008; Dilday et al. 2010).

Kronborg et al. (2010) inferred that the most magnified SN Ia in SNLS had a magnification factor $\mu=1.27$, corresponding to $\Delta m_{\mu}=2.5 \log _{10} \mu=0.26 \mathrm{mag}$, calculated from the location and photometry of foreground galaxies along the line of sight. Jönsson et al. (2010) came to a similar conclusion, finding that the SNLS sample did not probe SNe Ia with $\Delta m_{\mu} \gtrsim 0.25 \mathrm{mag}$ (however, see Karpenka et al. 2013, who find little to no lensing of the SNLS sample). Given the typical $\sim 0.2$ mag scatter in standardized SN Ia luminosities, a lensing signal at this level cannot be clearly ascertained for an individual object, although it can be detected statistically for a larger sample.

Lensing magnification by foreground galaxies is expected to play a larger role for more distant SNe. The Hubble Deep Field SN Ia 1997ff at $z \approx 1.7$ (Gilliland et al. 1999; Riess et al. 2001) is calculated to have a $\Delta m_{\mu}=0.34 \pm 0.12 \mathrm{mag}$ from lens galaxies projected nearby (Benítez et al. 2002). The uncertainties in the SN light curve and cosmological model preclude a definitive detection of the expected magnification signal. Rather, in this case the estimated lensing magnification has been applied as a correction to the inferred SN luminosity distance to constrain cosmological parameters, though care should be taken that this is done self-consistently (Zitrin et al. 2013).

Supernova properties were used by Quimby et al. (2013) to posit that the recent SN PS1-10afx at $z=1.39$ was an SN Ia magnified by a factor of $30\left(\Delta m_{\mu}=3.72 \pm 0.18 \mathrm{mag}\right)$ ! However, there is no lens observed in this system, and the authors conclude that it must be dark. Chornock et al. (2013) offer an alternate model, that this $\mathrm{SN}$ is best explained as a superluminous CC object, without significant magnification.

Ideally, we would like to confirm the detection of gravitational lensing magnification of an $\mathrm{SN}$ by independently checking the magnifications derived from the SN brightness and a lens-model prediction for consistency. In this paper, we discuss three gravitationally lensed SNe (SN CLO12Car, SN CLN12Did, and SN CLA11Tib) behind CLASH clusters. We present photometric observations of the $\mathrm{SNe}$ and spectroscopy of their host galaxies in Section 2. In Section 3, we discuss classification of the $\mathrm{SNe}$ and model light-curve fits. The gravitational magnifications from lens models are derived in Section 4, and we confront the models against the observations and discuss the results in Section 5. The following cosmological parameters are adopted throughout the paper (Sullivan et al. 2011): $H_{0}=71.6 \mathrm{~km} \mathrm{~s}^{-1}$ $\mathrm{Mpc}^{-1}, \Omega_{M}=0.27, \Omega_{\Lambda}=0.73$, and $\Omega_{k}=0$.

\section{OBSERVATIONS}

\subsection{SN CLO12Car}

SN CLO12Car was discovered in the CLASH images of MACSJ1720.2+3536 (Ebeling et al. 2010). The SN was detected in 2012 June with both ACS and WFC3/IR. Figure 1 shows the location of the SN $\left(\alpha=17^{\mathrm{h}} 20^{\mathrm{m}} 21^{\mathrm{s}} .03, \delta=+35^{\circ} 36^{\prime} 40^{\prime} \cdot 9\right.$, J2000), 0!'35 from the center of its host galaxy. The host is relatively bright in the IR $(\mathrm{F} 160 \mathrm{~W}=21.50 \pm 0.01 \mathrm{AB}$ mag $)$, but is significantly fainter in the optical images $(\mathrm{F} 606 \mathrm{~W}=$ $24.21 \pm 0.06 \mathrm{AB}$ mag). 
Table 1

SN CLO12Car Photometry

\begin{tabular}{|c|c|c|c|c|c|}
\hline UT Date & MJD & Instrument & Filter & $\begin{array}{c}\text { Exposure Time } \\
\text { (s) }\end{array}$ & Magnitude \\
\hline 2012 May 5 & 56052.58 & $\mathrm{ACS}$ & F850LP & 991.0 & $>25.546$ \\
\hline 2012 May 5 & 56052.64 & WFC3/IR & F160W & 1408.8 & $>25.347$ \\
\hline 2012 May 9 & 56056.02 & WFC3/IR & F105W & 1408.8 & $>26.016$ \\
\hline 2012 May 9 & 56056.04 & WFC3/IR & F140W & 1005.9 & $>25.808$ \\
\hline 2012 May 22 & 56069.68 & ACS & F814W & 1007.0 & $>26.666$ \\
\hline 2012 Jun 17 & 56095.66 & ACS & F850LP & 1032.0 & $24.597 \pm 0.130$ \\
\hline 2012 Jun 17 & 56095.68 & ACS & F814W & 975.0 & $25.148 \pm 0.082$ \\
\hline 2012 Jun 17 & 56095.73 & WFC3/IR & F160W & 1408.8 & $23.451 \pm 0.050$ \\
\hline 2012 Jun 17 & 56095.75 & WFC3/IR & F110W & 1005.9 & $23.856 \pm 0.024$ \\
\hline 2012 Jul 2 & 56110.09 & WFC3/IR & F105W & 1005.9 & $23.750 \pm 0.042$ \\
\hline $2012 \mathrm{Jul} 2$ & 56110.15 & WFC3/IR & F140W & 1005.9 & $23.271 \pm 0.025$ \\
\hline 2012 Jul 16 & 56124.09 & WFC3/IR & F105W & 1005.9 & $23.791 \pm 0.054$ \\
\hline 2012 Jul 16 & 56124.15 & WFC3/IR & F140W & 1005.9 & $23.250 \pm 0.025$ \\
\hline 2012 Jul 23 & 56131.31 & WFC3/IR & F160W & 455.9 & $23.340 \pm 0.063$ \\
\hline 2012 Jul 23 & 56131.38 & WFC3/IR & F105W & 455.9 & $24.138 \pm 0.056$ \\
\hline
\end{tabular}

Notes. All supernova photometry is reported in Vega magnitudes. The nondetections are reported as $3 \sigma$ upper limits.
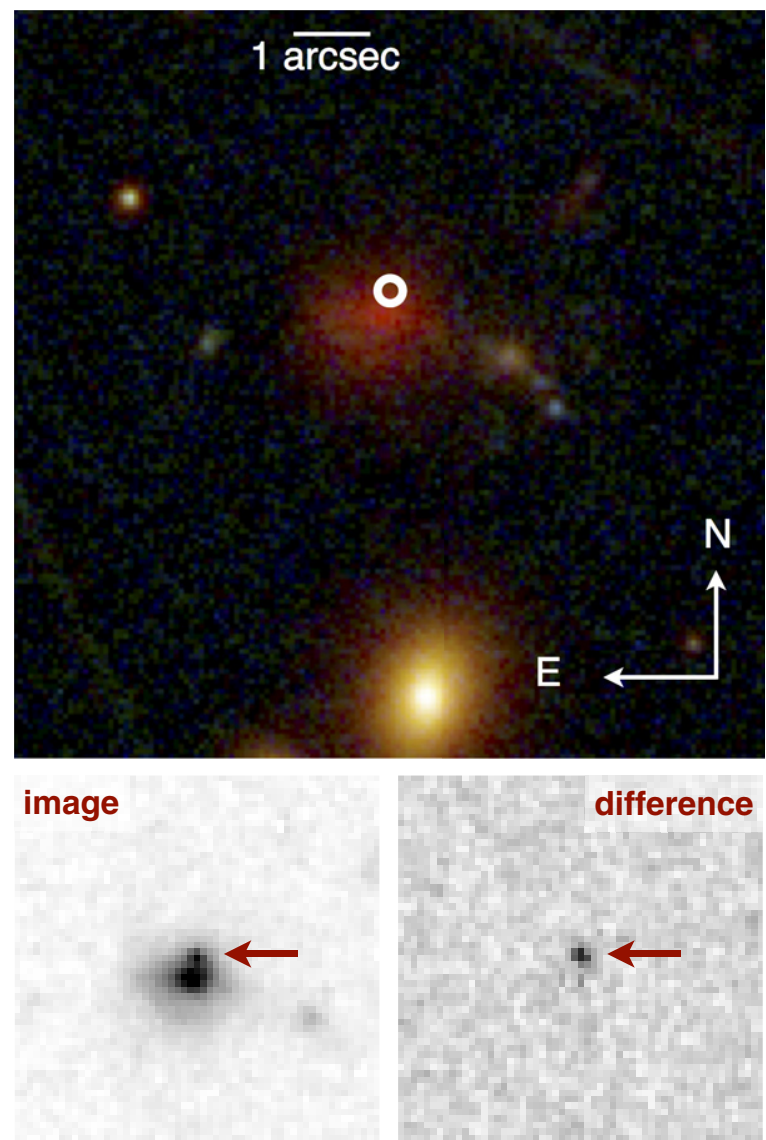

Figure 1. Top: CLASH HST composite false-color image of SN CLO12Car. In this image, the red channel is an average of the WFC3/IR F105W, F110W, F125W, F140W, and F160W data. The green channel combines ACS F606W, F625W, F775W, F814W, and F850LP observations, and the blue channel is from ACS F435W and F475W data. The location of the SN is marked with a white circle. Bottom: the left panel shows a $5^{\prime \prime}$ square cutout of the WFC3/IR F160W image from 2012 June 17, with SN CLO12Car marked by the arrow; the right panel shows the difference image after template subtraction.

(A color version of this figure is available in the online journal.)

We present aperture photometry for SN CLO12Car in Table 1 . These data include publicly available follow-up observations collected in 2012 July by HST program GO-12360
(PI: S. Perlmutter). The flux from SN CLO12Car was measured in difference images, using templates constructed with CLASH observations from 2012 March and April, more than 30 days before the first detection. For the ACS images, we used a fixed 4 pixel $(0.20)$ radius aperture, whereas for WFC3/IR, the photometry was measured in a 3 pixel radius $(0.27)$ aperture.

We used the DEep Imaging Multi-Object Spectrograph (DEIMOS; Faber et al. 2003) on the Keck-II 10 m Telescope to obtain a $\sim 2 \mathrm{hr}$ spectrum of the host galaxy of SN CLO12Car $(2 \times 1800 \mathrm{~s}, 1 \times 1700 \mathrm{~s}$, and $1 \times 1500 \mathrm{~s})$, with $\sim 4 \AA$ resolution, covering the wavelength range 5000-9000 $\AA$. The spectrum showed evidence of a weak emission line located between two night-sky lines near $8506 \AA$ A. For confirmation, we obtained a $2.5 \mathrm{hr}(5 \times 1800 \mathrm{~s})$ Gemini North Multi-Object Spectrograph (GMOS; Hook et al. 2004) nod-and-shuffle spectrum of the host, spanning 7460-9570 $\AA$ with a resolution of $\sim 2.1 \AA$. The GMOS spectrum also shows evidence for the faint line (marginally resolved as a doublet), which we identify as [O II] $\lambda \lambda 3726,3728$ emission at $z=1.281 \pm 0.001$. A composite of the DEIMOS and GMOS spectra is shown in the top panel of Figure 2. The spectroscopic redshift for the CLO12Car host galaxy lies near the peak of its photometric redshift (photo- $z$ ) probability density function (PDF), but the photo- $z$ PDF is broad, only limiting the redshift to $0.9 \lesssim z \lesssim 1.9$. A full description of the CLASH photo- $z$ estimation methods is given by Jouvel et al. (2014) and A. Molino et al. (in preparation). At $z=1.281$, a separation of 0.'35 from SN to host-galaxy center corresponds to a projected physical separation of $\sim 3 \mathrm{kpc}$ (modulo lensing magnification).

We also analyzed the follow-up HST WFC3/IR G102 and G141 grism (Dressel 2012) data for SN CLO12Car and its host galaxy (taken while the $\mathrm{SN}$ was still visible; program GO-12360, PI: S. Perlmutter). The G102 spectrum has a dispersion of $25 \AA$ pixel $^{-1}$, covering 8000-11500 $\AA$, while the G141 spectrum has a dispersion of $47 \AA$ pixel $^{-1}$, covering 11000-17000 $\AA$. The exposure time with each grating was about $1.3 \mathrm{hr}$, but this yielded only a weak signal, and we were unable to extract a clear SN spectrum. In these slitless grism observations, spectral features are effectively convolved with the spatial profile of the source, making sharp emission lines broader and less pronounced. There is an emission line in the G141 grism spectrum at $14963 \AA$, consistent with $\mathrm{H} \alpha \lambda 6563$ at $z \approx 1.28$, but this is only a marginal detection given the low resolution of the data. 


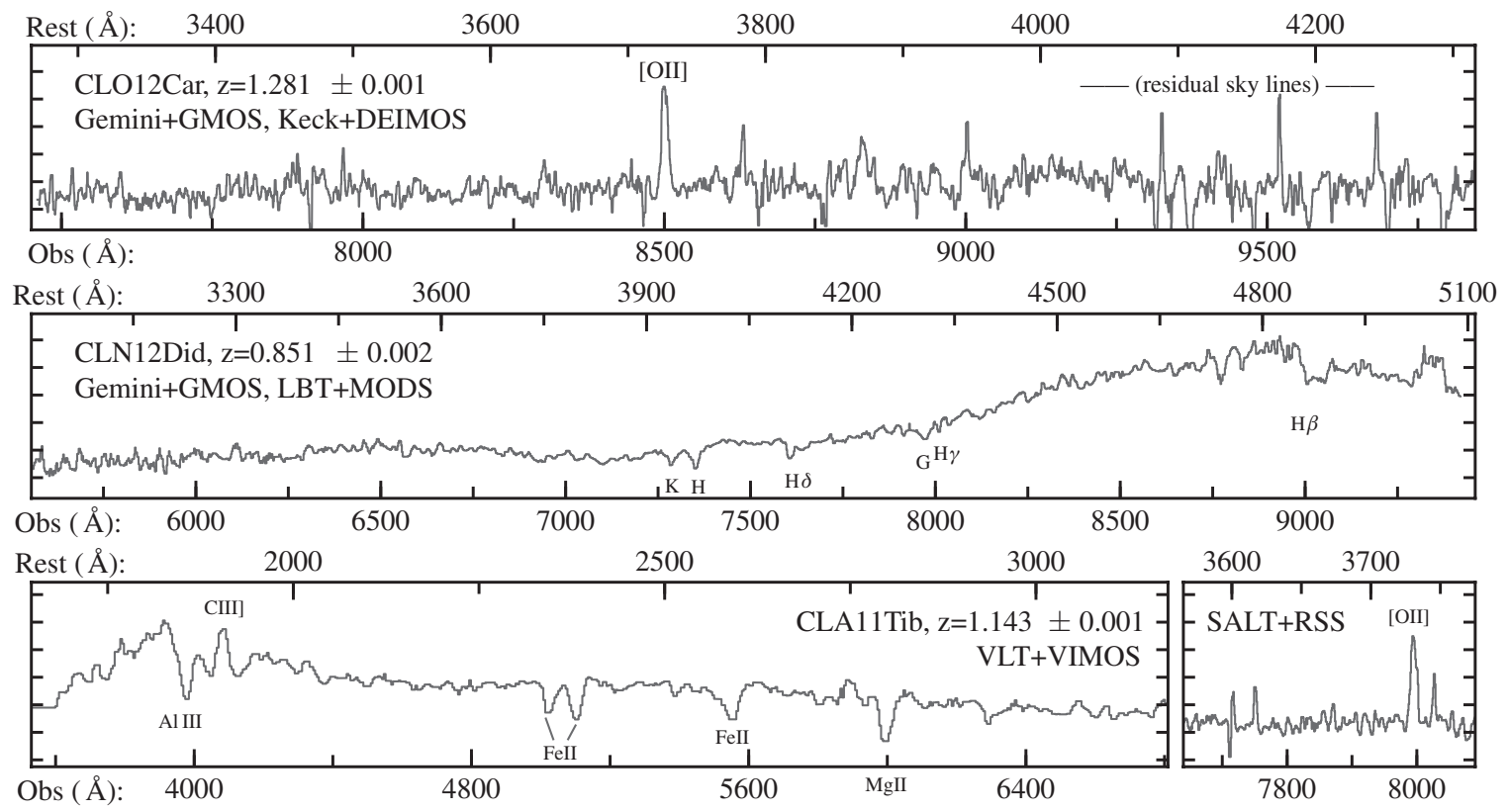

Figure 2. Spectra of the host galaxies of the three lensed SNe. Each panel shows observed wavelength along the bottom axis and rest wavelength on top. For display purposes, all spectra have been smoothed with a running 3 pixel median. Top: the host galaxy of SN CLO12Car, at $z=1.281 \pm 0.001$. The plot shows the mean spectrum combining observations with Gemini+GMOS and Keck+DEIMOS. The single emission line at $8500 \AA$ is identified as the [O II] $\lambda \lambda 3726,3728$ doublet based on the photo- $z$ and asymmetry of the line profile. Middle: the CLN12Did host galaxy, combining spectra from Gemini+GMOS and LBT+MODS. We derive $z=0.851 \pm 0.002$ from cross-correlation with galaxy templates and identification of $\mathrm{Ca}$ and $\mathrm{H}$ absorption features, as indicated. Bottom: the left panel shows the spectrum of the SN CLA11Tib host galaxy, observed with VLT+VIMOS. We derive $z=1.143 \pm 0.001$ from multiple absorption features and [C III] $\lambda 1909$ emission. This confirms the [O II] emission-line redshift from the SALT+RSS spectrum shown on the right.

Table 2

SN CLN12Did Photometry

\begin{tabular}{|c|c|c|c|c|c|}
\hline UT Date & MJD & Instrument & Filter & $\begin{array}{c}\text { Exposure Time } \\
\text { (s) }\end{array}$ & Magnitude \\
\hline 2012 Feb 3 & 55960.64 & ACS & F625W & 1032.0 & $26.699 \pm 0.171$ \\
\hline 2012 Feb 3 & 55960.65 & ACS & F850LP & 1017.0 & $25.066 \pm 0.150$ \\
\hline $2012 \mathrm{Feb} 18$ & 55975.67 & ACS & F775W & 1032.0 & $23.041 \pm 0.016$ \\
\hline $2012 \mathrm{Feb} 18$ & 55975.69 & ACS & F606W & 998.0 & $24.186 \pm 0.017$ \\
\hline 2012 Mar 3 & 55989.84 & ACS & F814W & 1032.0 & $23.175 \pm 0.015$ \\
\hline 2012 Mar 3 & 55989.86 & ACS & F435W & 1018.0 & $>28.560$ \\
\hline 2012 Mar 3 & 55990.24 & ACS & F625W & 1032.0 & $24.182 \pm 0.023$ \\
\hline 2012 Mar 3 & 55990.25 & ACS & F850LP & 1017.0 & $22.929 \pm 0.026$ \\
\hline 2012 Mar 3 & 55990.31 & ACS & F475W & 1032.0 & $27.057 \pm 0.138$ \\
\hline 2012 Mar 3 & 55990.32 & ACS & F775W & 1013.0 & $23.034 \pm 0.016$ \\
\hline 2012 Mar 3 & 55990.37 & WFC3/IR & F110W & 1508.8 & $22.915 \pm 0.013$ \\
\hline 2012 Mar 3 & 55990.39 & WFC3/IR & F160W & 1005.9 & $23.041 \pm 0.041$ \\
\hline 2012 Mar 16 & 56002.61 & ACS & F606W & 1032.0 & $25.491 \pm 0.038$ \\
\hline 2012 Mar 16 & 56002.62 & ACS & F814W & 984.0 & $23.410 \pm 0.018$ \\
\hline 2012 Mar 16 & 56002.67 & WFC3/IR & F105W & 1305.9 & $23.138 \pm 0.019$ \\
\hline 2012 Mar 16 & 56002.69 & WFC3/IR & F140W & 1305.9 & $23.540 \pm 0.034$ \\
\hline 2012 Mar 18 & 56004.74 & ACS & F475W & 1032.0 & $>28.924$ \\
\hline 2012 Mar 18 & 56004.75 & ACS & F850LP & 1001.0 & $23.359 \pm 0.037$ \\
\hline 2012 Mar 29 & 56015.52 & ACS & F435W & 1032.0 & $>28.625$ \\
\hline 2012 Mar 29 & 56015.53 & ACS & F814W & 1017.0 & $24.148 \pm 0.029$ \\
\hline 2012 Apr 12 & 56029.62 & ACS & F850LP & 1032.0 & $24.448 \pm 0.079$ \\
\hline 2012 Apr 12 & 56029.63 & ACS & F814W & 985.0 & $25.072 \pm 0.058$ \\
\hline 2012 Apr 12 & 56029.68 & WFC3/IR & F160W & 1508.8 & $23.316 \pm 0.038$ \\
\hline 2012 Apr 12 & 56029.70 & WFC3/IR & F110W & 1005.9 & $23.853 \pm 0.038$ \\
\hline
\end{tabular}

Notes. All SN photometry is reported in Vega magnitudes. The nondetections are listed as $3 \sigma$ upper limits.

\subsection{SN CLN12Did}

SN CLN12Did was discovered in early 2012 in CLASH ACS and WFC3/IR images of RXJ1532.9+3021 (Ebeling et al. 1998). Figure 3 shows the position of the SN (located at $\alpha=15^{\mathrm{h}} 32^{\mathrm{m}} 59^{\mathrm{s}} .25, \delta=+30^{\circ} 21^{\prime} 42^{\prime \prime} .8, \mathrm{~J} 2000$ ), and we present aperture photometry of the SN in Table 2 . The SN was present in the first epoch of observations in each of the ACS and WFC3/IR filters, so we did not have an $\mathrm{SN}$-free template for subtraction. Nonetheless, the $\mathrm{SN}$ is separated by $\sim 4^{\prime \prime}$ from its putative host 

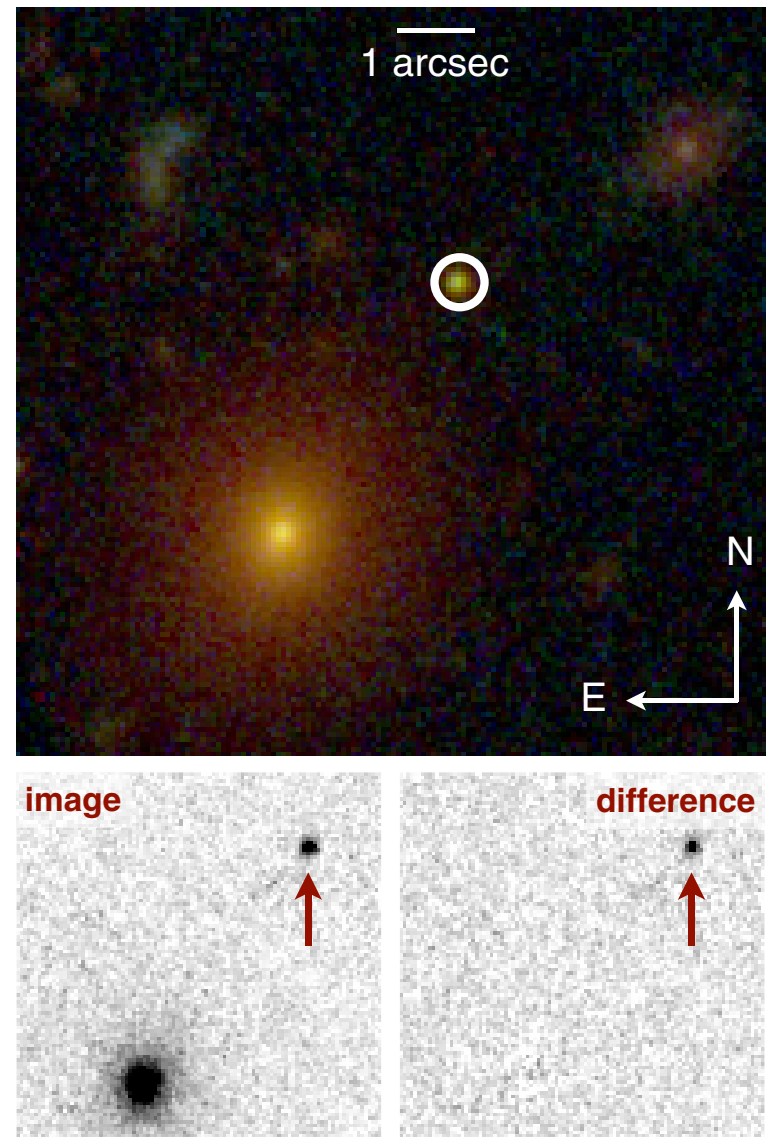

Figure 3. Top: HST composite false-color image of SN CLN12Did and surroundings. The red channel is an average of WFC3/IR F105W, F110W, $\mathrm{F} 140 \mathrm{~W}$, and $\mathrm{F} 160 \mathrm{~W}$ data from the CLASH program, the green channel combines the ACS F606W, F625W, F775W, F814W, and F850LP observations, and the blue channel is from ACS F435W and F475W data. The SN position is marked with a white circle, in the outskirts of its early-type host galaxy to the southeast. Bottom: the left panel shows a 5" square cutout of the ACS F850LP image from 2012 March 3, with SN CLN12Did marked by the arrow; the right panel shows the difference image after template subtraction.

(A color version of this figure is available in the online journal.)

galaxy, so we expect negligible host-galaxy contamination in the SN photometry.

Ground-based Sloan Digital Sky Survey (SDSS) DR7 images (Abazajian et al. 2009) of the CLN12Did host, along with the HST data, show the CLN12Did host to be an early-type, likely elliptical, galaxy. The CLASH data yield photometry for the host galaxy of $\mathrm{F} 606 \mathrm{~W}=22.69 \pm 0.03 \mathrm{AB}$ mag and $\mathrm{F} 160 \mathrm{~W}=$ $19.434 \pm 0.003 \mathrm{AB}$ mag. We obtained a $\sim 5 \mathrm{hr}$ spectrum $(6 \times 2700 \mathrm{~s}$ and $1 \times 2500 \mathrm{~s})$ of the host galaxy using the MultiObject Double Spectrographs (MODS; Pogge et al. 2010) on the Large Binocular Telescope (LBT), covering 6300-9000 with 15-40 A resolution. We also used Gemini North GMOS to obtain a $4.5 \mathrm{hr}(9 \times 1800 \mathrm{~s})$ spectrum of the galaxy, covering 5220-9420 ̊, with a resolution of $\sim 6 \AA$. A composite spectrum from the Gemini and LBT observations is shown in the middle panel of Figure 2. We derived respective redshifts of $0.851 \pm$ 0.001 and $0.852 \pm 0.002$ from these data, via cross-correlation with absorption-line templates. This redshift is consistent with the photo- $z$ derived for the galaxy $\left(z_{\text {phot }}=0.92_{-0.07}^{+0.05}\right)$. We adopt $z=0.851 \pm 0.001$ for SN CLN12Did. At this redshift, the 4 " separation from SN to host-galaxy center corresponds to a projected physical separation of $\sim 30 \mathrm{kpc}$ (modulo lensing magnification).

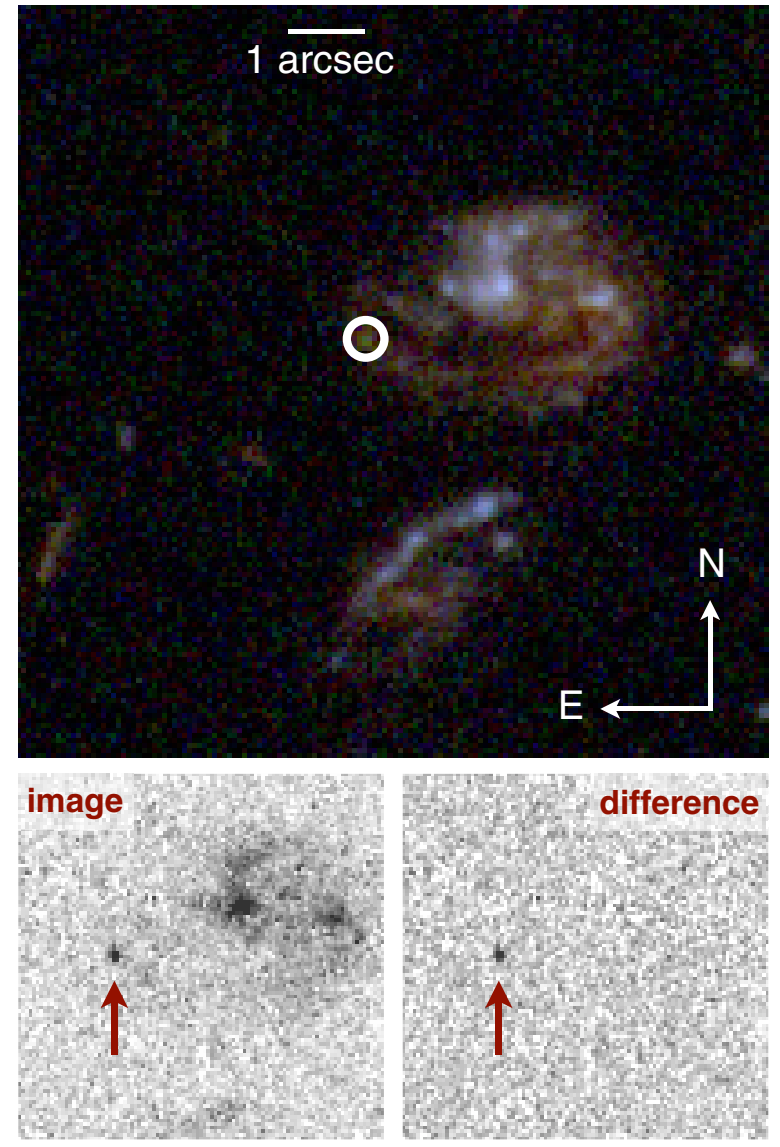

Figure 4. Top: HST composite false-color image of SN CLA11Tib from CLASH data. The red channel is an average of WFC3/IR F105W, F110W, F125W, F140W, and F160W data. The green channel combines ACS F606W, F625W, F775W, F814W, and F850LP observations, and the blue channel is from ACS F435W and F475W data. The location of the SN is marked with a white circle. Bottom: the left panel shows a 5" square cutout of the ACS F850LP image taken on UT 2011 January 4, with SN CLA11Tib indicated by the arrow; the right panel shows the difference image after template subtraction.

(A color version of this figure is available in the online journal.)

\subsection{SN CLA11Tib}

SN CLA11Tib was discovered in CLASH ACS and WFC3/ IR images of A383 (Abell et al. 1989) in 2011 January. Figure 4 shows the SN in a color image (located at $\alpha=02^{\mathrm{h}} 48^{\mathrm{m}} 01^{\mathrm{s}} .27$, $\delta=-03^{\circ} 33^{\prime} 16^{\prime \prime}$. 9 , J2000). Aperture photometry for the ACS observations of SN CLA11Tib is presented in Table 3. The WFC3/IR observations of the SN were taken as part of the HST follow-up program GO-12360 (PI: S. Perlmutter). The ACS photometry is from difference images with host-galaxy subtraction, but no template images are available for the WFC3/IR observations (F105W, F125W, and F160W), so the reported IR photometry is based on the direct images. As the $\mathrm{SN}$ is located in a spiral arm (see Figure 4), the host galaxy could contaminate aperture photometry for these observations. For this reason we report point-spread function (PSF) fitting photometry of the SN in the WFC3/IR observations from Dolphot (a modified version of HSTphot; Dolphin 2000). Photometry of the host galaxy gives $\mathrm{F} 606 \mathrm{~W}=22.88 \pm 0.01 \mathrm{AB}$ mag and $\mathrm{F} 160 \mathrm{~W}=21.51 \pm$ $0.01 \mathrm{AB}$ mag.

We obtained Southern African Large Telescope (SALT) + Robert Stobie Spectrograph (RSS; Nordsieck 2012; Crawford et al. 2010) spectroscopy of the host of SN CLA11Tib with a 1200 s exposure using the PG0900 grating (range 6300-9300 , 
Table 3

SN CLA11Tib Photometry

\begin{tabular}{|c|c|c|c|c|c|}
\hline UT Date & MJD & Instrument & Filter & $\begin{array}{c}\text { Exposure Time } \\
\text { (s) }\end{array}$ & Magnitude \\
\hline 2010 Nov 18 & 55518.91 & ACS & F625W & 1032.0 & $>27.206$ \\
\hline 2010 Nov 18 & 55518.92 & ACS & F850LP & 1014.0 & $>25.768$ \\
\hline 2010 Nov 18 & 55518.99 & ACS & F775W & 1010.0 & $>26.792$ \\
\hline 2010 Dec 8 & 55538.41 & ACS & F850LP & 1032.0 & $>25.802$ \\
\hline 2010 Dec 8 & 55538.43 & ACS & F814W & 1060.0 & $>26.872$ \\
\hline $2010 \operatorname{Dec} 28$ & 55558.45 & ACS & $\mathrm{F} 435 \mathrm{~W}$ & 1032.0 & $>27.385$ \\
\hline $2010 \operatorname{Dec} 28$ & 55558.46 & ACS & F814W & 1092.0 & $24.699 \pm 0.049$ \\
\hline 2011 Jan 4 & 55565.97 & ACS & F625W & 1032.0 & $25.349 \pm 0.074$ \\
\hline 2011 Jan 4 & 55565.98 & ACS & F850LP & 1092.0 & $24.251 \pm 0.091$ \\
\hline 2011 Jan 18 & 55579.35 & ACS & F606W & 1032.0 & $26.364 \pm 0.104$ \\
\hline 2011 Jan 18 & 55579.36 & ACS & F814W & 1059.0 & $24.394 \pm 0.038$ \\
\hline 2011 Jan 22 & 55583.41 & ACS & F775W & 1032.0 & $24.762 \pm 0.066$ \\
\hline 2011 Jan 22 & 55583.43 & ACS & F606W & 1073.0 & $26.551 \pm 0.127$ \\
\hline 2011 Jan 24 & 55585.08 & WFC3/IR & F105W & 806.0 & $23.755 \pm 0.037$ \\
\hline 2011 Jan 24 & 55585.09 & WFC3/IR & F125W & 806.0 & $23.402 \pm 0.036$ \\
\hline 2011 Jan 24 & 55585.14 & WFC3/IR & F160W & 906.0 & $23.357 \pm 0.059$ \\
\hline $2011 \mathrm{Feb} 7$ & 55599.38 & ACS & F814W & 1032.0 & $25.082 \pm 0.076$ \\
\hline 2011 Feb 7 & 55599.40 & ACS & $\mathrm{F} 435 \mathrm{~W}$ & 1093.0 & $>27.336$ \\
\hline $2011 \mathrm{Feb} 21$ & 55613.16 & ACS & F850LP & 1994.0 & $25.247 \pm 0.188$ \\
\hline 2011 Mar 1 & 55621.43 & ACS & F850LP & 1076.0 & $>25.677$ \\
\hline
\end{tabular}

Notes. All SN photometry is reported in Vega magnitudes. The nondetections are listed as $3 \sigma$ upper limits. Dolphot was used to derive PSF photometry for the WFC3/IR observations (see text).

resolution $6 \AA$ ), and detect a strong emission line (but close to a night-sky line) that we identify as [O II] at $z=1.144 \pm 0.001$. We confirmed this identification with the VIsible MultiObject Spectrograph (VIMOS; Le Fèvre et al. 2003) on the Very Large Telescope (VLT) observations in a $\sim 4 \mathrm{hr}$ spectrum of the host galaxy (range 3900-6700 ^ with $28 \AA$ resolution). The spectrum shows Mg II and Fe II absorption, and [C III] $\lambda 1909$ emission, all at a consistent redshift of $1.143 \pm 0.001$, which we adopt for SN CLA11Tib. This redshift is consistent with the photo- $z$ derived for the galaxy $\left(z_{\text {phot }}=1.05 \pm 0.10\right)$. At this redshift, SN CLA11Tib is projected $\sim 25 \mathrm{kpc}$ from the center of its host galaxy (uncorrected for lensing magnification).

\section{SUPERNOVA CLASSIFICATION AND LIGHT-CURVE FITS}

Classification of high-redshift $(z \gtrsim 1)$ SNe is challenging (e.g., Rodney et al. 2012; Rubin et al. 2013; Jones et al. 2013). For example, a typical SN Ia has a peak luminosity in the restframe $B$ band of $M_{B} \approx-19.4 \mathrm{mag}$. At $z=1.5$ this becomes a $Y$-band apparent magnitude of $\sim 25.8$. Key spectral absorption features at rest-frame optical wavelengths (such as the Si II feature observed in SN Ia at $\sim 6150 \AA$ ) are redshifted into the IR, making them inaccessible to most ground-based spectrographs. With these constraints, definitive spectroscopic classification of high- $z \mathrm{SNe}$ requires prohibitively large investments of HST time (Jones et al. 2013).

For this reason, we rely on photometric classifications for these three lensed SNe. We utilize a Bayesian photometric SN classifier called STARDUST: Supernova Taxonomy And Redshift Determination Using SuperNova ANAlysis (SNANA) Templates. An early version of this classifier was first introduced by Jones et al. (2013), and the complete version used for this work is presented in more detail by Graur et al. (2014) and S. A. Rodney et al. (in preparation). In brief, observations are compared against simulated $\mathrm{SNe}$ Ia and $\mathrm{CC} \mathrm{SNe}$, gener- ated using version $10.27 \mathrm{k}$ of the SNANA software package ${ }^{36}$ (Kessler et al. 2009b). For SNe Ia the STARDUST classifier uses the SALT2 light-curve model (Guy et al. 2010) extended to cover the rest-frame near-UV and IR wavelengths (see below), whereas for CC SNe, the classifier draws on the SNANA library of $16 \mathrm{SN} \mathrm{Ib/c} \mathrm{and} 26 \mathrm{SN}$ II templates, to be described in further detail by Sako et al. (2014). An important capability of STARDUST that we employ for this work is to allow an achromatic flux scaling factor for every model realization. This means that our STARDUST classifications rely only on the observed SN colors and light-curve shape, and do not depend on the intrinsic SN luminosities or our choice of cosmological model. This is especially important for fitting lensed $\mathrm{SNe}$, as it allows us to classify the SNe independent of the lensing magnification.

For additional verification of our photometric SN classifications, we turned to the Photometric SuperNova IDentification software (PSNID; Sako et al. 2008, 2011) to classify the three SNe. PSNID is similar to the STARDUST classifier, as both use SALT2 to simulate SNe Ia and rely on the same $16 \mathrm{SN} \mathrm{Ib/c} \mathrm{and}$ 26 SN II templates to simulate CC SNe. However, PSNID has the advantage that it has been thoroughly tested, obtaining the highest figure of merit in the SN photometric classification challenge (Kessler et al. 2010), and it is already publicly available as a component of SNANA. A downside of PSNID for this work is that it uses absolute magnitude priors in its classifications, which is not ideal for analyzing lensed SNe.

For SNe Ia, we also employed a modified version of the Guy et al. (2010) SALT2 model to fit light curves and derive standardized luminosity distances. The version of SALT2 presented by Guy et al. (2010) has been robustly tested, and was used in SNLS cosmology papers (Sullivan et al. 2011; Conley et al. 2011). To accommodate the wide wavelength range of our data, we extended the SALT2 model to cover rest-frame near-UV and IR wavelengths (by default, the SALT2 fitter can only fit rest

\footnotetext{
$36 \mathrm{http} / / / \mathrm{sdssdp} 62 . \mathrm{fnal.gov/sdsssn/SNANA-PUBLIC/}$
} 
wavelengths of 2800-7000 ̊). For all three SNe, this extended SALT2 model produces similar results (within $1 \sigma$ ) to those of the original Guy et al. (2010) model. In deriving distances, we converted our SALT2 parameters representing light-curve shape and color excess ( $x 1$ and $c$ ) to their respective SiFTO (Conley et al. 2008) parameters ( $s$ and $C$ ), using the conversion equations from Guy et al. (2010). This was done in order to adopt the constants $M, \alpha$, and $\beta$, from Sullivan et al. (2011) and also used by Jones et al. (2013). Specifically, the distance modulus was calculated using the following equation: ${ }^{37}$

$$
\mathrm{dm}_{\mathrm{SALT} 2}=m_{B}^{*}-M+\alpha(s-1)-\beta C,
$$

with $M=-19.12 \pm 0.03, \alpha=1.367 \pm 0.086$, and $\beta=$ $3.179 \pm 0.101$. To match the (for our purposes, arbitrary) normalization of the SALT2 fitter used by Guy et al. (2010) to our results from SNANA, we applied an offset of 0.27 mag to the value of $m_{B}^{*}$ reported by SNANA.

We also used the Jha et al. (2007) version of MLCS2k2 included in SNANA to fit the SNe Ia. To check for consistency, we compared the best-fit light-curve shape parameter $\Delta$ from MLCS2k 2 to $x 1$ from SALT 2 using the relationship in Appendix G given by Kessler et al. (2009a). To compare the SALT2 color parameter $c$ with MLCS2k2 $A_{V}$, we employ a linear relationship derived from Figure 42 of Kessler et al. (2009a), specifically

$$
c=(0.464 \pm 0.021) A_{V}-(0.121 \pm 0.014) .
$$

Finally, to ensure a consistent zeropoint between the SALT2 and MLCS2k2 distance moduli, we applied both methods to a sample of SDSS Supernova Survey SNe Ia from Holtzman et al. (2008) and Kessler et al. (2009a). We added a zeropoint correction of $0.20 \mathrm{mag}$ to the distance moduli reported by SNANA in the MLCS2k2 fit $\left(\mathrm{dm}_{\mathrm{MLCS} 2 \mathrm{k} 2}\right)$, to put them on the same scale as the SALT2 distances $\left(\mathrm{dm}_{\mathrm{SALT} 2}\right)$.

Although we report magnitudes in Tables 1-3, both the classification and SN Ia light-curve fitting were done in flux space. The upper limits for each SN were included in the fits with the measured flux and $1 \sigma$ uncertainties.

\subsection{SN CLO12Car}

SN CLO12Car was located in a red disky galaxy, in which both SNe Ia and CC SNe could be expected. The STARDUST classifier prefers an SN Ia fit with probability $\left(P_{\mathrm{Ia}}\right)$ of 0.91 . The probabilities for $\mathrm{SN} \mathrm{Ib/c}\left(P_{\mathrm{Ib} / \mathrm{c}}\right)$ and SN II $\left(P_{\mathrm{II}}\right)$ models are $10^{-8}$ and 0.09 , respectively. The best-fit $\mathrm{SN} \mathrm{Ia,} \mathrm{SN} \mathrm{Ib/c,}$ and SN II models had $\chi^{2} / v(v=11)$ of $1.12,4.56$, and 1.76, respectively. PSNID also favors an SN Ia fit at a high confidence, with $P_{\mathrm{Ia}}=0.99$ and a best-fit $\chi^{2} / v=1.06(v=11)^{38}$. We thus conclude that SN CLO12Car was an SN Ia.

Both MLCS2k2 and SALT2 produce good light-curve fits for SN CLO12Car. We show the SALT2 light-curve fit along with the $1 \sigma$ model errors in Figure 5. The SALT2 fit had a $\chi^{2} / v=$ $13.3 / 11=1.21$, and the fit parameters $(x 1=1.375 \pm 0.829$ and $c=0.254 \pm 0.049$ ) are in the range of normal SNe Ia (Guy et al. 2010). From these parameters, we derive a distance modulus of $\mathrm{dm}_{\mathrm{SALT} 2}=43.83 \pm 0.24 \mathrm{mag}$ for the SN.

\footnotetext{
37 We use the notation "dm" for distance modulus, rather than the conventional $\mu$, to avoid confusion with the lensing magnification $\mu$. As usual, it is defined as $\mathrm{dm}=5 \log _{10} d_{L}+25$, where $d_{L}$ is the luminosity distance in Mpc.

38 PSNID uses a luminosity prior, as discussed above. Correcting the light curve for lensing, assuming reasonable values of magnification between 1 mag of demagnification and 3 mag of magnification, changes the $P_{\text {Ia }}$ value slightly (ranging from 0.87 to 0.99 ).
}

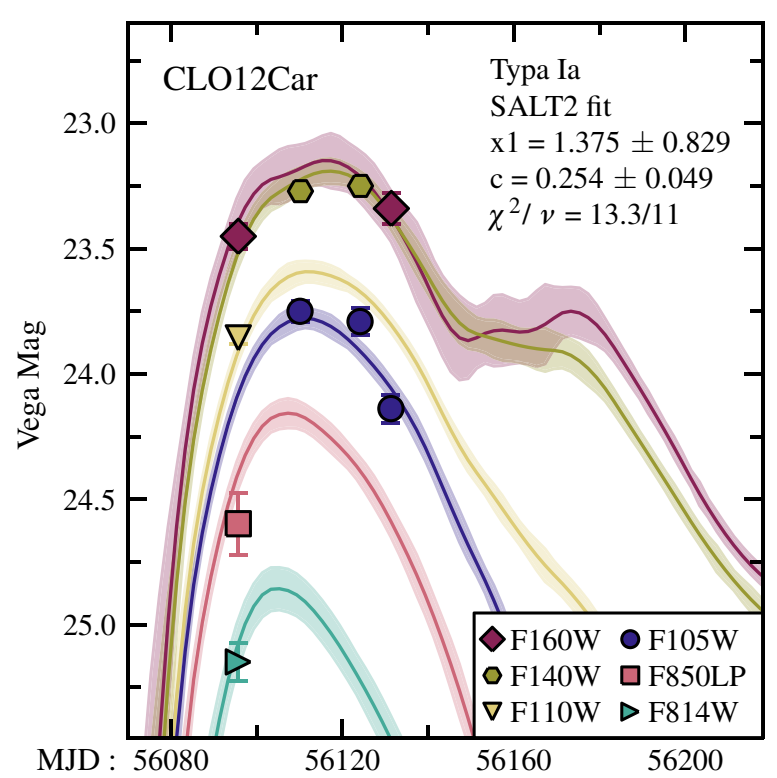

Figure 5. SALT2 light-curve fit of SN CLO12Car. The light-curve parameters $x 1$ and $c$ are typical of normal SNe Ia, and the data are well matched by the model, with $\chi^{2} / v=13.3 / 11$.

(A color version of this figure is available in the online journal.)

The MLCS2k2 light-curve fit had a slightly worse, but still acceptable, goodness-of-fit $\left(\chi^{2} / v=18.1 / 11=1.64\right)$. The model parameters were $\Delta=0.007 \pm 0.314$ and $A_{V}=0.635 \pm$ $0.190 \mathrm{mag}$. The light-curve shape parameters from the two fitters agree at $<1 \sigma$; specifically, the SALT2 $x 1$ value corresponds to $\Delta=-0.380 \pm 0.160$. The best-fit MLCS2k $2 A_{V}$ corresponds to $c=0.174 \pm 0.090$, which is also $<1 \sigma$ from the best-fit SALT2 color. Moreover, we find that $\mathrm{dm}_{\mathrm{MLCS} 2 \mathrm{k} 2}=43.71 \pm 0.16 \mathrm{mag}$ (after applying the zeropoint correction), again consistent with the inferred dm $\mathrm{dALT}_{2}$ at $<1 \sigma$.

For comparison, at $z=1.281$, the distance modulus expected from $\Lambda \mathrm{CDM}$ with our adopted cosmological parameters is $\mathrm{dm}_{\Lambda \mathrm{CDM}}=44.76 \mathrm{mag}$, significantly higher than what is measured from the SN light curve. We discuss the implications of this finding in Section 4.

\subsection{SN CLN12Did}

SN CLN12Did was located in an elliptical galaxy, already indicating it was most likely an SN Ia (Cappellaro et al. 1999; Mannucci 2005; Foley \& Mandel 2013). The STARDUST classifier prefers an SN Ia fit at high confidence $(>5 \sigma)$. PSNID also favors an SN Ia fit with high significance $\left(P_{\text {Ia }}>0.9999\right)$, with best-fit $\chi^{2} / v=0.5(v=20)$. These consistent results yield the strong conclusion that SN CLN12Did was an SN Ia.

We show the SALT2 SN Ia light-curve fit for SN CLN12Did in Figure 6. Similar to Figure 5, we display the light-curve points and the model curves with $1 \sigma$ errors. Upper limits on $\mathrm{F} 435 \mathrm{~W}$ and $\mathrm{F} 475 \mathrm{~W}$ nondetections are excluded from the plot for clarity, but all of the observations listed in Table 2 are used in the lightcurve fit. The SALT2 parameters fall within the normal SN Ia range, with $x 1=0.383 \pm 0.208, c=-0.042 \pm 0.021$, and $\chi^{2} / v=28.5 / 20=1.42$. From the light-curve parameters, we derive $\mathrm{dm}_{\mathrm{SALT} 2}=43.33 \pm 0.15 \mathrm{mag}$.

MLCS2k2 produces a slightly better light-curve fit, with $\chi^{2} / v=23.6 / 20=1.18$. The best-fit parameters are $A_{V}=$ $0.042 \pm 0.059 \mathrm{mag}$ and $\Delta=0.055 \pm 0.098$. Using the conversion equations, the MLCS2k2 fit $A_{V}$ corresponds to 

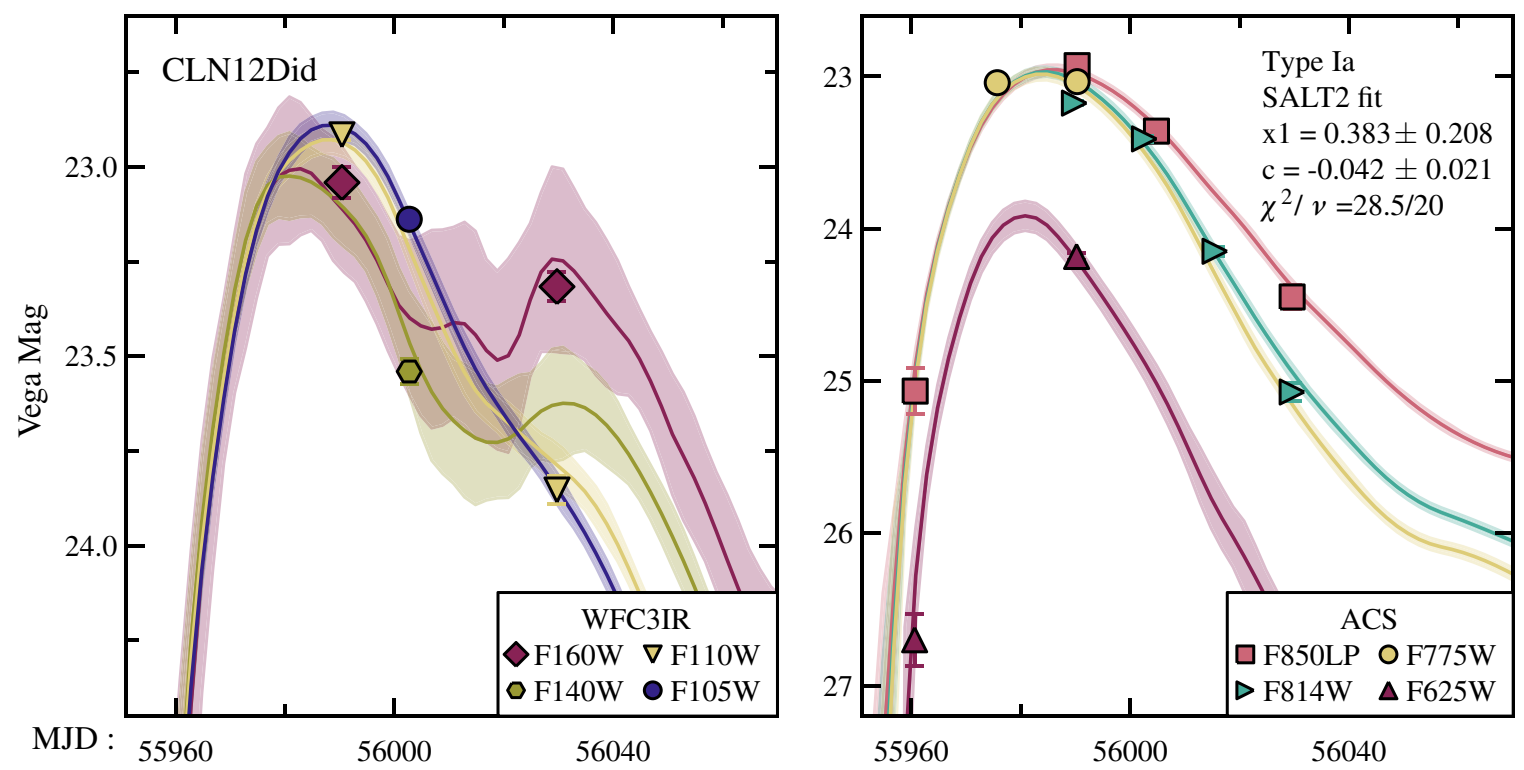

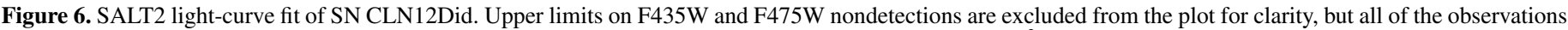

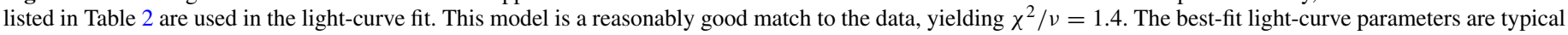
for a normal SN Ia.

(A color version of this figure is available in the online journal.)

SALT2 $c=-0.102 \pm 0.031$, and the SALT2 $x 1$ predicts $\operatorname{MLCS} 2 \mathrm{k} 2 \Delta=-0.212 \pm 0.040$. This is good agreement in the color parameter, but a slight $(\sim 2.5 \sigma)$ discrepancy in light-curve shape. The inferred distance is $\mathrm{dm}_{\mathrm{MLCS} 2 \mathrm{k} 2}=43.28 \pm 0.09 \mathrm{mag}$, consistent with the SALT2 result. For comparison, at $z=0.851$, the cosmological parameters yield $\mathrm{dm}_{\Lambda \mathrm{CDM}}=43.65 \mathrm{mag}$.

\subsection{SN CLA11Tib}

SN CLN12Tib is located in a spiral arm of its blue, late-type host galaxy, an environment in which all SN types can be found (Cappellaro et al. 1999; Mannucci 2005; Li et al. 2011; Foley \& Mandel 2013). The STARDUST classifier prefers a CC fit for this $\mathrm{SN}$, returning probabilities $P_{\mathrm{II}}=0.41$ and $P_{\mathrm{Ib} / \mathrm{c}}=0.52$, compared to the SN Ia fit probability $P_{\mathrm{Ia}}=0.07$.

We show the light-curve fit for SN CLA11Tib in Figure 7, with each row giving the maximum-likelihood fit for a single SN subclass. The $\chi^{2} / v(v=14)$ values for the best-fit SN Ia, SN $\mathrm{Ib} / \mathrm{c}$, and SN II models are 3.81, 3.42, and 3.55, respectively. None of the three SN types are a good fit to the light curve; moreover, none of the templates provide a good fit to all the WFC3/IR data. There are only $42 \mathrm{CC}$ SNe templates, and we do not expect them to represent the full range of SNe II and SNe Ibc. The SN II template that provides the best match to the observations is SDSS-018793 (SN 2007og), an SN IIP

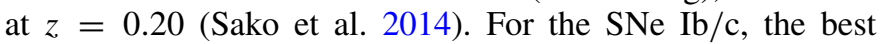
match comes from SN SDSS-017548 (SN 2007ms), an SN Ic at $z=0.0393$ (Östman et al. 2011). The sole HST WFC3/IR F125W observation of SN CLA11Tib is close to the peak for both the SN IIP and SN Ic models; at $z=1.143$, the F125W filter corresponds to approximately the rest $V$ band. To further highlight the difficulty in photometrically classifying SN CLA11Tib, in Figure 8 we show color-color diagrams from the ACS and WFC3/IR photometry taken during 2011 January 22-24, where the SN straddles the typical colors of SNe Ia, Ib/c, and II, though favoring CC SN models. The full STARDUST classification uses these types of constraints with all available epochs.
Our PSNID results disagree with those from the STARDUST classifier. PSNID favors an SN Ia fit for SN CLA11Tib at greater than $4 \sigma$. However, the best-fit PSNID SN Ia model does not match the light curve well $\left(\chi^{2} / v=2.83\right.$, with $v=$ 14). To check these results, we fit SN CLA11Tib with SALT2 and MLCS2k2 directly using SNANA. The best SALT2 fit has normal light-curve parameters, with $x 1=0.114 \pm 0.486$ and $c=0.048 \pm 0.037$, yielding $\mathrm{dm}_{\mathrm{SALT} 2}=44.07 \pm 0.19 \mathrm{mag}$ (for comparison, $\mathrm{dm}_{\Lambda \mathrm{CDM}}=44.45 \mathrm{mag}$ ). However, as with PSNID, the SNANA SALT2 fit requires an unacceptably large $\chi^{2} / v=$ $57.8 / 14=4.13$. These results do not change substantially if we use the restricted-wavelength version of SALT2; the fit remains poor. The MLCS2k2 results were much better $\left(\chi^{2} / v=29.8 / 14=2.13\right)$, with best-fit light-curve parameters of $A_{V}=0.389 \pm 0.193 \mathrm{mag}, \Delta=-0.011 \pm 0.244$, and $\mathrm{dm}_{\text {MLCS2k2 }}=43.97 \pm 0.14$ mag. Using the conversion equations, the MLCS2k2 fit $A_{V}$ corresponds to SALT2 $c=0.059 \pm 0.091$, and the SALT2 $x 1$ predicts MLCS2k2 $\Delta=-0.201 \pm 0.093$. Interestingly, the MLCS2k2 and SALT2 parameters are similar, but the SALT2 fit is significantly worse.

To summarize, our classification analysis of SN CLA11Tib is inconclusive. The light curve was best fit by CC SN templates with STARDUST, while PSNID preferred an SN Ia fit. Similar to SN CLO12Car, we corrected the light curve for a varying amount of lensing (from 1 mag of demagnification to 3 mag of magnification) and fit with PSNID to see if the luminosity prior was driving the classification. The value of $P_{\text {Ia }}$ varied greatly, with the probability dropping to 0 with magnifications $>1.5$ mag. Because of these conflicting results, we further explore both CC SN and SN Ia models in our lensing analysis of SN CLA11Tib (Section 4.3).

\section{GRAVITATIONAL LENSING MAGNIFICATION}

In this section, we derive the magnification of each $\mathrm{SN}$ in two independent ways. First, we use the SNe themselves, from the observed SN brightness and light-curve fits described in Section 3. Second, we independently determine the expected 

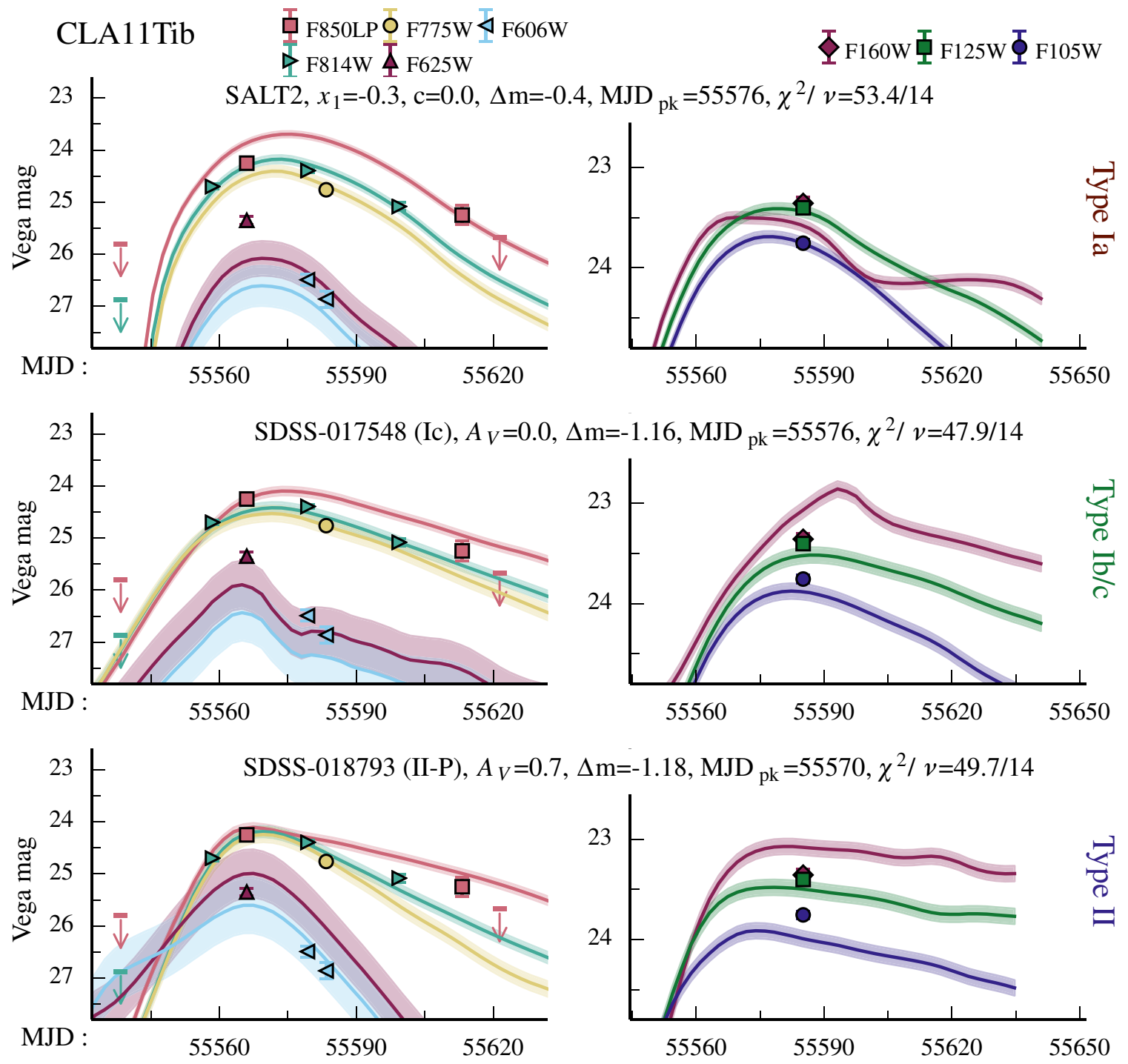

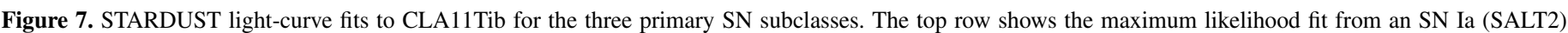

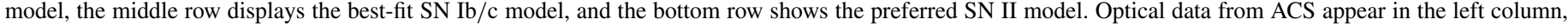

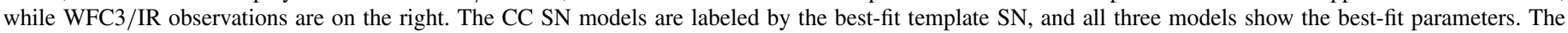

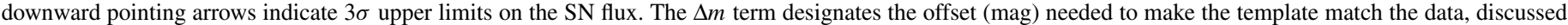
further in Section 4.3.

(A color version of this figure is available in the online journal.)

magnification from cluster mass maps derived by the CLASH team from strong+weak-lensing features.

One approach to inferring the SN magnification is simply measuring the offset between the inferred distance modulus and that predicted by the cosmological model $\left(\mathrm{dm}_{\Lambda \mathrm{CDM}}\right)$. In that case, however, statistical and systematic uncertainties in the cosmological parameters (which are themselves derived in part from SNe Ia over a wide redshift range) need to be included in the analysis. A more direct approach is to compare the CLASH SNe directly to a "field" sample at similar redshifts. For a sufficiently small redshift range, the relation between distance modulus and redshift can be taken to be linear. The field sample of SNe Ia define the zeropoint and slope of that relationship, and we derive the inferred magnification of our SN Ia relative to that locus, with all of these SNe analyzed in an identical way. This differential approach is insensitive to zeropoint offsets between the fitters and cosmological parameters, and only makes the assumption that all $\mathrm{SNe}$ Ia at nearly the same redshift are consistently standardizable (so it is less susceptible to evolution or other SN Ia systematics that vary with redshift).

For the predicted magnification from the CLASH cluster mass maps, we analyzed both the strong- and weak-lensing features in the clusters. The identification of multiple images in the cluster fields was done using the 16-band HST imaging obtained in the context of the CLASH campaign. The Zitrin et al. (2009) method revealed nine multiple image systems in the case of A383 and seven systems in MACS J1720. Unfortunately, no multiple-image systems were found in the field of RX J1532, the cluster with the lowest total mass in this study. Where available, spectroscopic redshifts from the literature or the CLASH-VLT program (Balestra et al. 2013) determined the distance to the strong-lensing features. The reliable 16-band photometric redshifts (Jouvel et al. 2014) from the CLASH pipeline delivered the redshifts of systems without spectroscopic confirmation. In the case of weak lensing, we used 5-band CLASH Subaru imaging to derive weak-lensing shear 


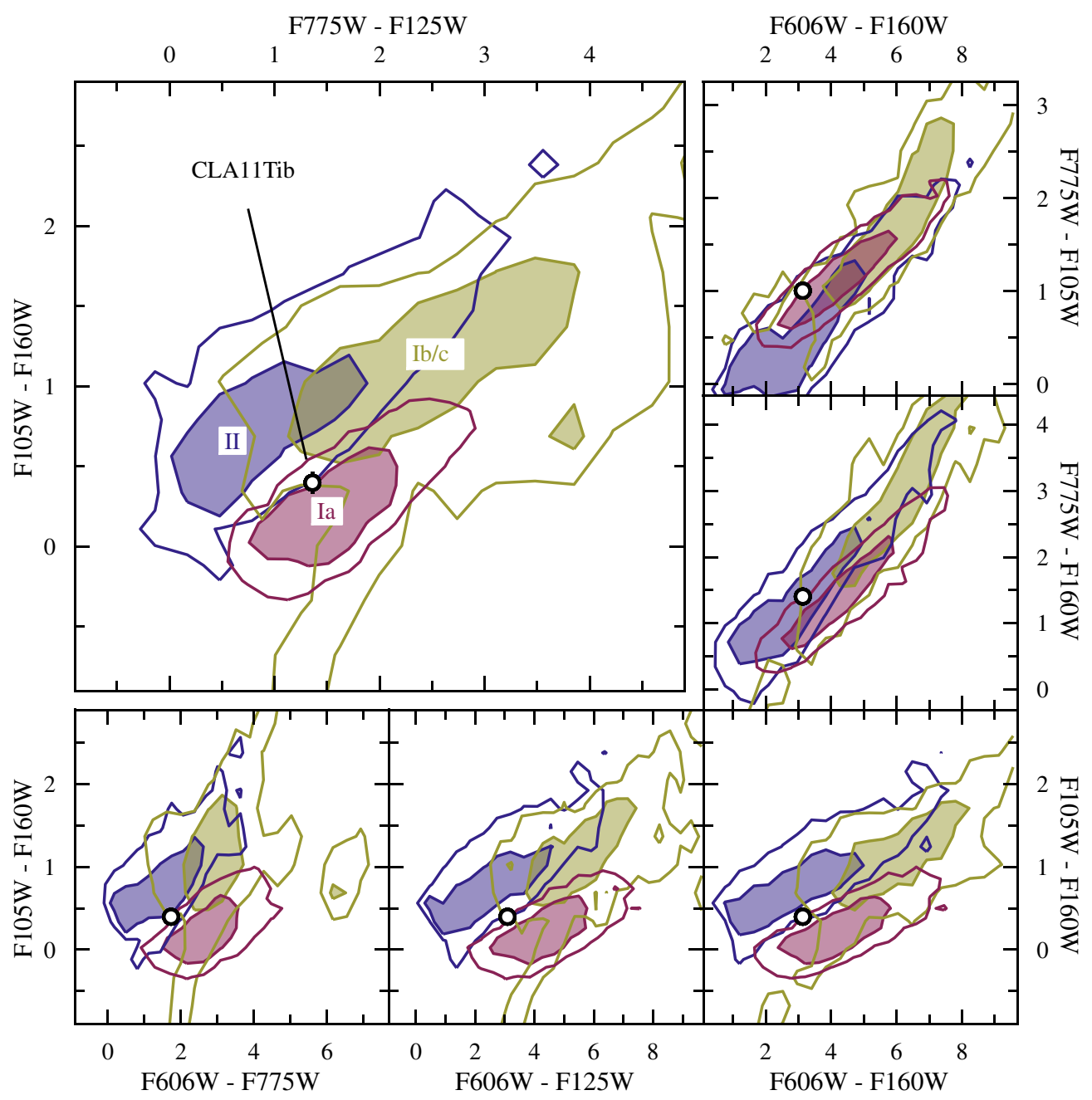

Figure 8. Color-color diagrams for SN CLA11Tib photometry from MJD 55583-55585, illustrating the difficulty in photometric classification of this object. We utilize color-based classification so as to be unaffected by achromatic gravitational lensing. The data favor a CC origin for this SN (either SN Ib/c or SN II). The contours shown are derived from SNANA Monte Carlo simulations with 5000 simulated SNe for each of the three subclasses (so they do not reflect the relative frequency across classes). Shaded regions enclose $68 \%$ of each population and solid lines enclose $95 \%$.

(A color version of this figure is available in the online journal.)

catalogs. The underlying pipeline for image reduction, shape measurement, and background selection is thoroughly outlined by Umetsu et al. (2012) and Medezinski et al. (2013).

To combine the constraints from the weak and strong-lensing regime into a consistent lens model, we used SaWLens (Merten et al. 2009, 2011; Meneghetti et al. 2010; Umetsu et al. 2012; Medezinski et al. 2013). This nonparametric method also reconstructs the mass distribution in the regime far away from the strong-lensing features, or where no strong-lensing features are available, and both cases apply in our study. By nonparametric, we mean that no a priori assumptions on the mass distribution of the lens were made. The method reconstructs the lensing potential, the rescaled and line-of-sight integrated counterpart of the Newtonian potential, on an adaptively refined mesh using both weak- and strong-lensing inputs. In the case of RX J1532, where no strong-lensing features were found, we use only the shear catalogs to constrain the lens mass distribution. From the lensing potential maps we directly derive the magnification (e.g., Bartelmann 2010) at the redshift of interest and read off its value at the position of each $\mathrm{SN}$, as described individually below. In order to assign error bars to these values, we run 1500 realizations of each lens model by
Table 4

Comparing SN Magnifications with Lensing Predictions

\begin{tabular}{lccc}
\hline \hline SN Name & \multicolumn{2}{c}{ Lensing from Light-curve Fits } & \\
\cline { 2 - 3 } & $\begin{array}{c}\Delta m_{\mu} \\
\text { (SALT2) }\end{array}$ & $\begin{array}{c}\Delta m_{\mu} \\
\text { (MLCS2k2) }\end{array}$ & $\begin{array}{c}\Delta m_{\mu} \\
\text { (Lensing Maps) }\end{array}$ \\
\hline SN CLO12Car & $0.91 \pm 0.25$ & $1.06 \pm 0.17$ & $0.83 \pm 0.16$ \\
SN CLN12Did & $0.24 \pm 0.15$ & $0.15 \pm 0.09$ & $0.28 \pm 0.08$ \\
SN CLA11Tib & $\ldots$ & $\ldots$ & $0.43 \pm 0.11$
\end{tabular}

Notes. Comparison of lensing predictions from light-curve fits and lensing maps for the three SNe. All of the $\Delta m_{\mu}$ table entries have units of magnitudes. For both SN CLO12Car and SN CLN12Did, there is good agreement (within $1 \sigma$ ) between both lensing methods. The classification of SN CLA11Tib was inconclusive, making it difficult to determine a magnification from the light curve (see Section 4.3).

bootstrapping the weak-lensing input catalogs and randomly sampling through the allowed redshift error range of each strong-lensing feature (Merten et al. 2011).

The independent magnification estimates, from both methods above, are summarized in Table 4 . We consider each object 


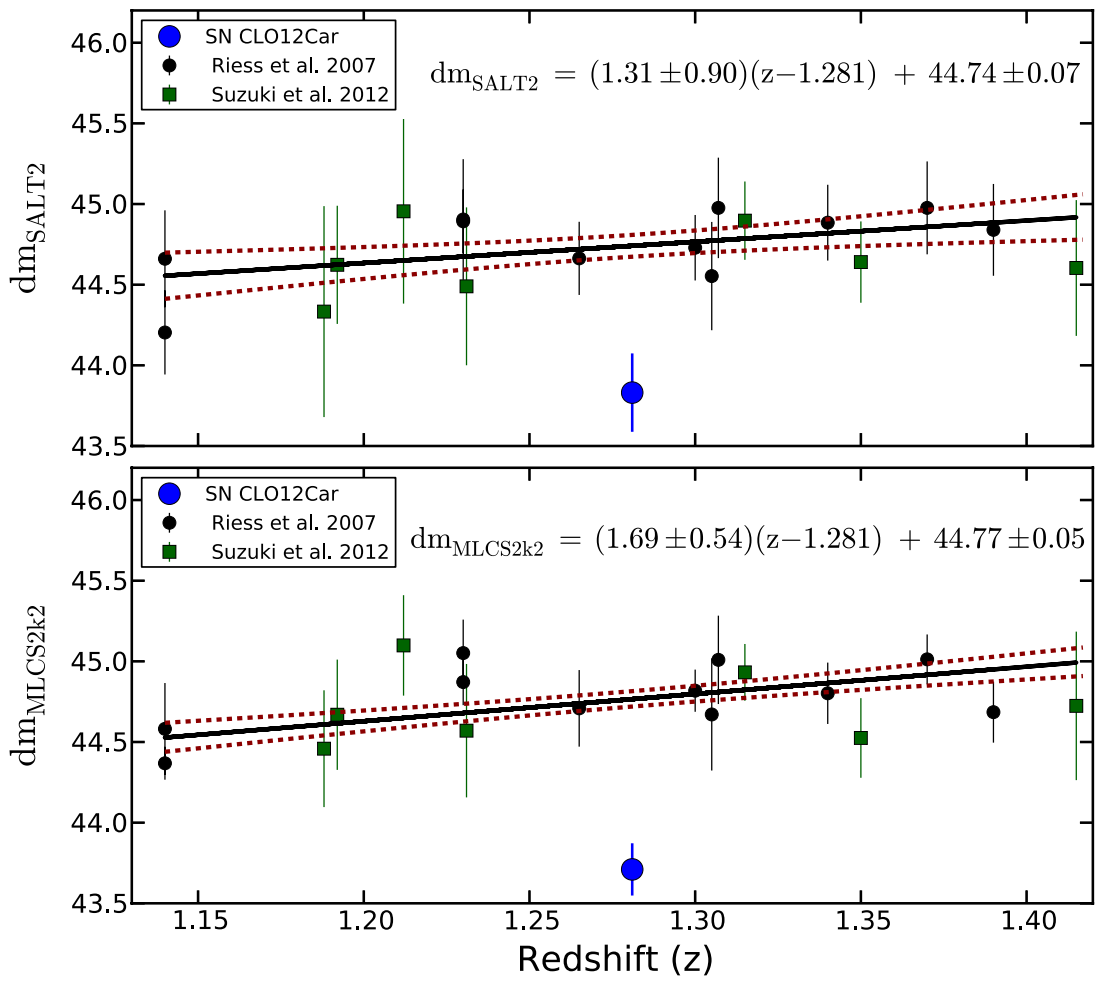

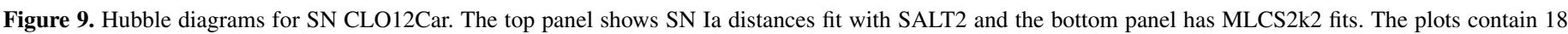

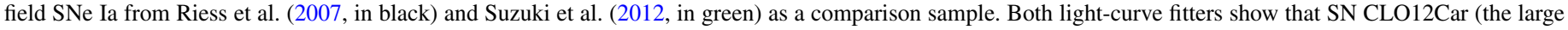

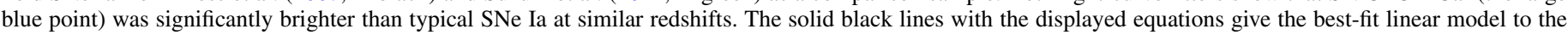

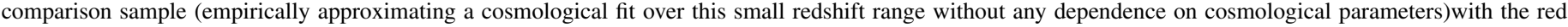

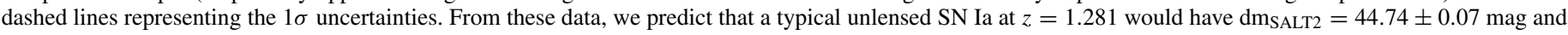

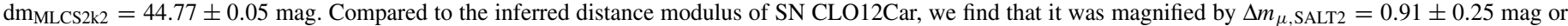
$\Delta m_{\mu, \text { MLCS2k2 }}=1.06 \pm 0.17 \mathrm{mag}$. Both light-curve fitters give a consistent and significant magnification (greater than unity)

(A color version of this figure is available in the online journal.)

individually below. Because we could not conclusively classify SN CLA11Tib, we consider both the SN Ia and CC SN models for it (Section 4.3).

\subsection{SN CLO12Car}

We showed in Sections 2.1 and 3.1 that SN CLO12Car was an SN Ia at $z=1.281$. In Figure 9, we display two Hubble diagrams for the SN. The top plot contains SN Ia distances fit with SALT2 and the bottom plot with MLCS2k2. Both plots also show a comparison sample of 18 field SNe Ia from Riess et al. (2007) and Suzuki et al. (2012) in the range $1.14 \leqslant z \leqslant 1.41$, fit in exactly the same manner as SN CLO12Car. Consistently with either light-curve fitter, we find that SN CLO12Car was significantly brighter than the typical $\mathrm{SNe}$ Ia at a similar redshift. The solid black line in both panels represents the best linear fit to the field SN Ia sample, with the fit slope and intercept as shown. ${ }^{39}$ From this analysis, we can estimate the lensing magnification as determined by the SN itself. The linear fits to the field sample predict $\operatorname{dm}_{\text {SALT2 }}(z=1.281)=$ $44.74 \pm 0.07 \mathrm{mag}$ and $\operatorname{dm}_{\mathrm{MLCS} 2 \mathrm{k} 2}(z=1.281)=44.77 \pm 0.05$ mag. With these values, we derive that SN CLO12Car was magnified by $\Delta m_{\mu, \mathrm{SALT} 2}=0.91 \pm 0.25 \mathrm{mag}$ (corresponding to a magnification $\mu_{\text {SALT2 }}=2.31 \pm 0.54$ ) in the SALT2 model,

\footnotetext{
39 Three of the Suzuki et al. (2012) SNe Ia plotted in Figure 9 were also behind galaxy clusters and potentially lensed. However, the object with the highest estimated magnification still only has $\mu=1.07$, corresponding to $\Delta m_{\mu}=0.07 \mathrm{mag}$, well within the SN Ia scatter. Removing these three SNe Ia from the comparison sample has a negligible effect on our results.
}

or $\Delta m_{\mu, \mathrm{MLCS} 2 \mathrm{k} 2}=1.06 \pm 0.17 \mathrm{mag}\left(\mu_{\mathrm{MLCS} 2 \mathrm{k} 2}=2.65 \pm 0.42\right)$ with MLCS2k2.

The cluster lensing magnification map (derived as described above) around MACS J1720 for a source at the SN redshift $(z=$ 1.281 ) is shown in Figure 10. From the map, we derive a lensing magnification for SN CLO12Car of $\mu=2.15 \pm 0.33$, which corresponds to $\Delta m_{\mu}=0.83 \pm 0.16 \mathrm{mag}$. The magnification from the light-curve fits and the lensing maps agree to well within $1 \sigma$. Moreover, SN CLO12Car is inconsistent with being unlensed; its SN Ia fit requires $\Delta m_{\mu}>0$ at $\sim 4 \sigma$.

\subsection{SN CLN12Did}

We showed in Sections 2.2 and 3.2 that SN CLN12Did was an SN Ia at $z=0.851$. In Figure 11, we display two Hubble diagrams for the SN, mirroring the analysis for SN CLO12Car (see Section 4.1 and Figure 9). The comparison field sample contains $63 \mathrm{SNe}$ Ia from the SNLS sample (Guy et al. 2010) in the range $0.75 \leqslant z \leqslant 0.95$. Unlike the case for SN CLO12Car, we see that SN CLN12Did falls well within the normal scatter for typical SNe Ia in the redshift range. Both light-curve fitters do find SN CLN12Did to be brighter than expected compared to the field sample, but not significantly so $(\sim 1 \sigma)$. Our inferred magnification estimates for SN CLN12Did are $\Delta m_{\mu, \text { SALT2 }}=0.24 \pm 0.15 \mathrm{mag}\left(\mu_{\mathrm{SALT} 2}=1.25 \pm 0.18\right)$ and $\Delta m_{\mu, \mathrm{MLCS} 2 \mathrm{k} 2}=0.15 \pm 0.09 \mathrm{mag}\left(\mu_{\mathrm{MLCS} 2 \mathrm{k} 2}=1.15 \pm 0.10\right)$.

The cluster lensing magnification map around RX J1532 for a source at $z=0.851$ is shown in Figure 12. It predicts a lensing magnification for SN CLN12Did of $\Delta m_{\mu}=0.28 \pm 0.08 \mathrm{mag}$ 


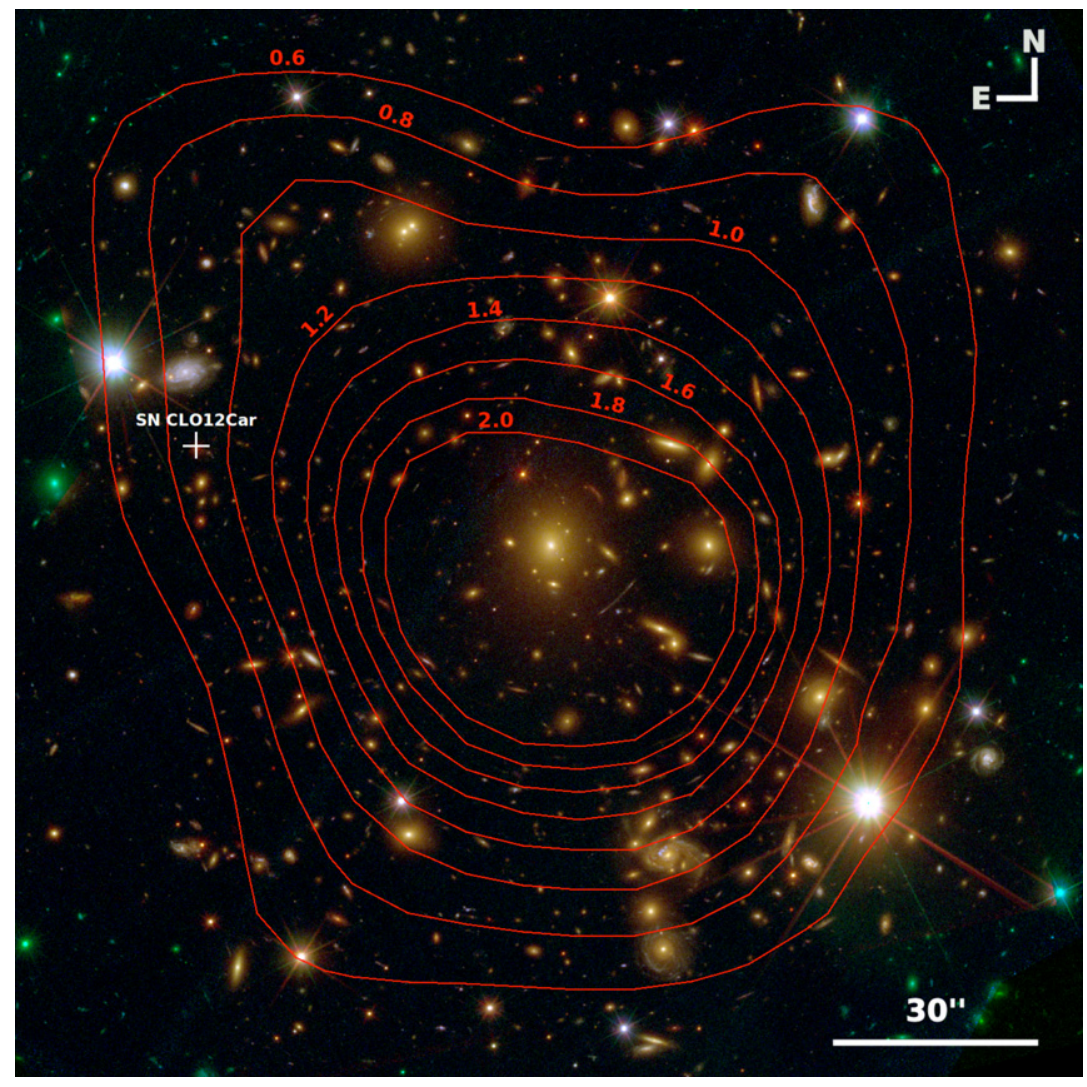

Figure 10. Magnification map for SN CLO12Car $(z=1.281)$ behind MACSJ1720.2 + $3536(z=0.391)$. The image shows an RGB false-color background based on 12-band $H S T$ /CLASH optical and near-IR images of the cluster field. The location of the SN is marked with a white cross. The contours show the magnitude increase induced by the lensing magnification $\left(\Delta m_{\mu}=2.5 \log _{10} \mu\right)$ of the cluster for a source at the SN redshift. The lensing magnification was derived from weakand strong-lensing constraints jointly, and computed using the SaWLens lensing reconstruction algorithm (Merten et al. 2009, 2011). Multiple images used for the strong-lensing constraints in this system will be presented by A. Zitrin et al. (in preparation).

(A color version of this figure is available in the online journal.)
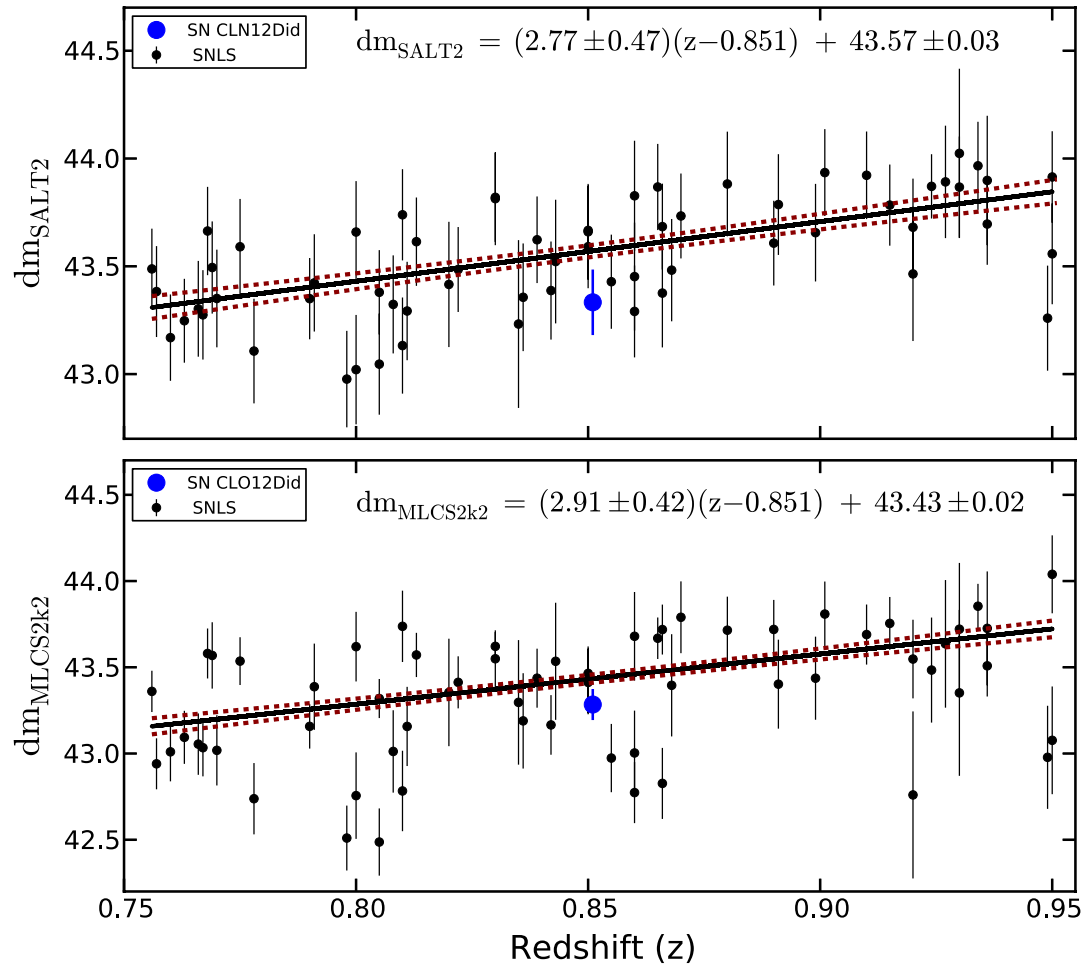

Figure 11. Hubble diagrams for SN CLN12Did. As in Figure 9, the top and bottom panels show SALT2 and MLCS2k2 fits, respectively. The field comparison sample consists of 63 SNe Ia from SNLS (Guy et al. 2010). Both light-curve fitters suggest slight (but insignificant) magnification for the SN, with $\Delta m_{\mu, \mathrm{SALT} 2}=0.24 \pm 0.15$ $\mathrm{mag}$ and $\Delta m_{\mu, \mathrm{MLCS} 2 \mathrm{k} 2}=0.15 \pm 0.09 \mathrm{mag}$.

(A color version of this figure is available in the online journal.) 


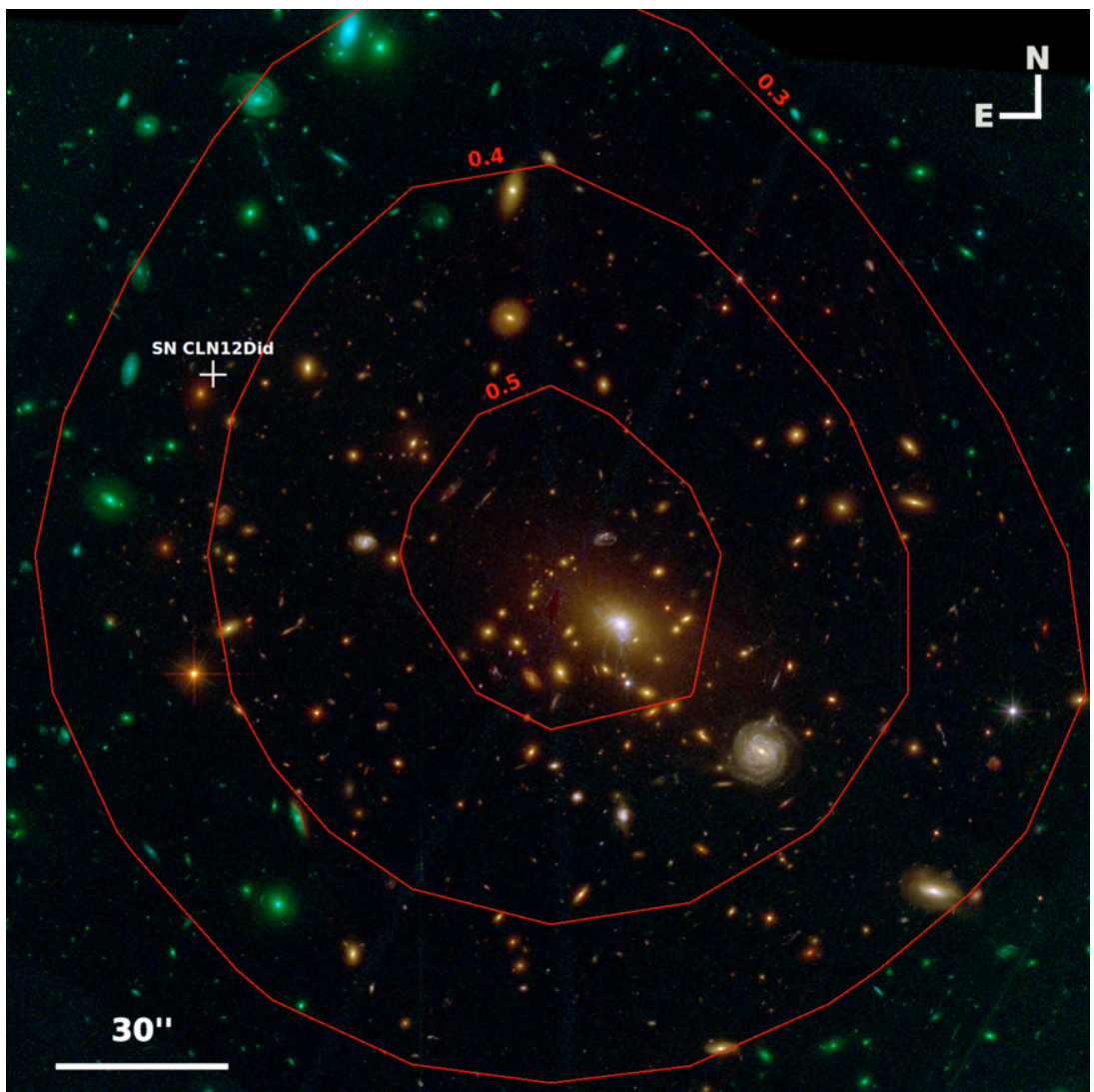

Figure 12. Magnification map for SN CLN12Did $(z=0.851)$ behind RXJ1532.9 $3021(z=0.345)$. Similar to Figure 10, the color image is based on CLASH optical and near-IR images, with the SN location marked by a white cross and lensing magnification ( $\Delta m_{\mu}$, in mag units) shown with red contours. Because RX J1532 is a much weaker lensing cluster than either MACS J1720 or A383, this map was derived using weak-lensing constraints alone, computed with the SaWLens algorithm (Merten et al. 2009, 2011). The resolution of the reconstructed map from weak-lensing constraints is much poorer than for the other two clusters, so the displayed contours are heavily smoothed, and the Monte Carlo magnification estimate at the SN position is actually $\Delta m_{\mu}=0.28 \pm 0.08 \mathrm{mag}$.

(A color version of this figure is available in the online journal.)

$(\mu=1.29 \pm 0.09)$. Just like in the case of SN CLO12Car, the lensing map prediction is consistent with the magnification inferred from the SN light curve, though for SN CLN12Did the magnification is only detected at low significance.

\subsection{SN CLA11Tib}

We were unable to classify SN CLA11Tib conclusively (see Section 3.3), so we take a slightly different approach to the analysis for this object. Figure 13 shows the predicted lensing magnification map for a source at the redshift of SN CLA11Tib $(z=1.143)$ behind A383. From the map, we derive a lensing magnification for the $\mathrm{SN}$ of $\mu=1.48 \pm 0.15$, which corresponds to $\Delta m_{\mu}=0.43 \pm 0.11 \mathrm{mag}$.

If we assume that SN CLA11Tib was an SN Ia, repeating the analysis above (as for SN CLO12Car and SN CLN12Did) yields magnification estimates for SN CLA11Tib of $\Delta m_{\mu, \mathrm{SALT} 2}=$ $0.52 \pm 0.20 \mathrm{mag}$ and $\Delta m_{\mu, \mathrm{MLCS} 2 \mathrm{k} 2}=0.64 \pm 0.15 \mathrm{mag}$. However, as mentioned in Section 3.3, the SALT2 light-curve fit is unacceptable, with $\chi^{2} / v=57.8 / 14=4.13$, making the inferred distance unreliable. The MLCS2k2 light-curve fit $\left(\chi^{2} / v=29.8 / 14=2.13\right)$ is much better, and agrees well with the lensing map prediction (within $1 \sigma$ ). However, due to the discrepancy of the goodness of fit between MLCS2k2 and SALT2, we hesitate to definitively classify the SN as an SN Ia.

To compare both SN Ia and CC SN models in the lensing analysis for SN CLA11Tib, we show in Figure 14 the goodness-of-fit for both sets of models. The plot gives the best-fit $\mathrm{SN}$ Ia results as above, as well as $\mathrm{SN}$ II and $\mathrm{SN} \mathrm{Ib/}$ c templates (restricted to those with $\chi^{2} / v<5$ ). For the CC SN templates, STARDUST calculates a magnitude offset $\Delta m$ (shown also in Figure 7) that is required for the best match of the template to the data, and we show this on the abscissa of Figure 14.

We found that the best-fitting CC SN models (albeit at $\chi^{2} / v>3$ ) from STARDUST were the SN Ic SDSS-017548 and the SN IIP SDSS-018793. The SN Ic and SN IIP models had best-fit model $m_{J}=23.52 \pm 0.08 \mathrm{mag}$ and $m_{J}=$ $23.48 \pm 0.08 \mathrm{mag}$, respectively. The observed red color of SN CLA11Tib matches the SN Ic model with no relative extinction, but the SN II model requires $A_{V}=0.7 \mathrm{mag}$. Including this, we find the unextinguished absolute magnitudes of the models are $M_{R}=-19.34 \pm 0.08 \mathrm{mag}$ (SN Ic) or $M_{R}=-20.43 \pm 0.08 \mathrm{mag}$ (SN IIP). These are quite bright for $\mathrm{CC} \mathrm{SNe,} \mathrm{though} \mathrm{some}$ of this could be a result of the lensing magnification. The peak $M_{R}$ required by both models is much brighter than the original templates; the SN SDSS-017548 and SN SDSS-018793 spectral energy distributions have $M_{R}=-18.00 \mathrm{mag}$ and $M_{R}=-18.55 \mathrm{mag}$, respectively. The offset between the template and data ( $\Delta m$; Figures 7 and 14$)$ is $-1.16 \mathrm{mag}$ for the SN Ic model and -1.18 mag for the SN IIP model. These would be our best estimates for the lensing magnification based on the SN light curve, but an abundance of caution is warranted, as these estimates assume that SN CLA11Tib had the same intrinsic luminosity as the best-fit template, a dubious 


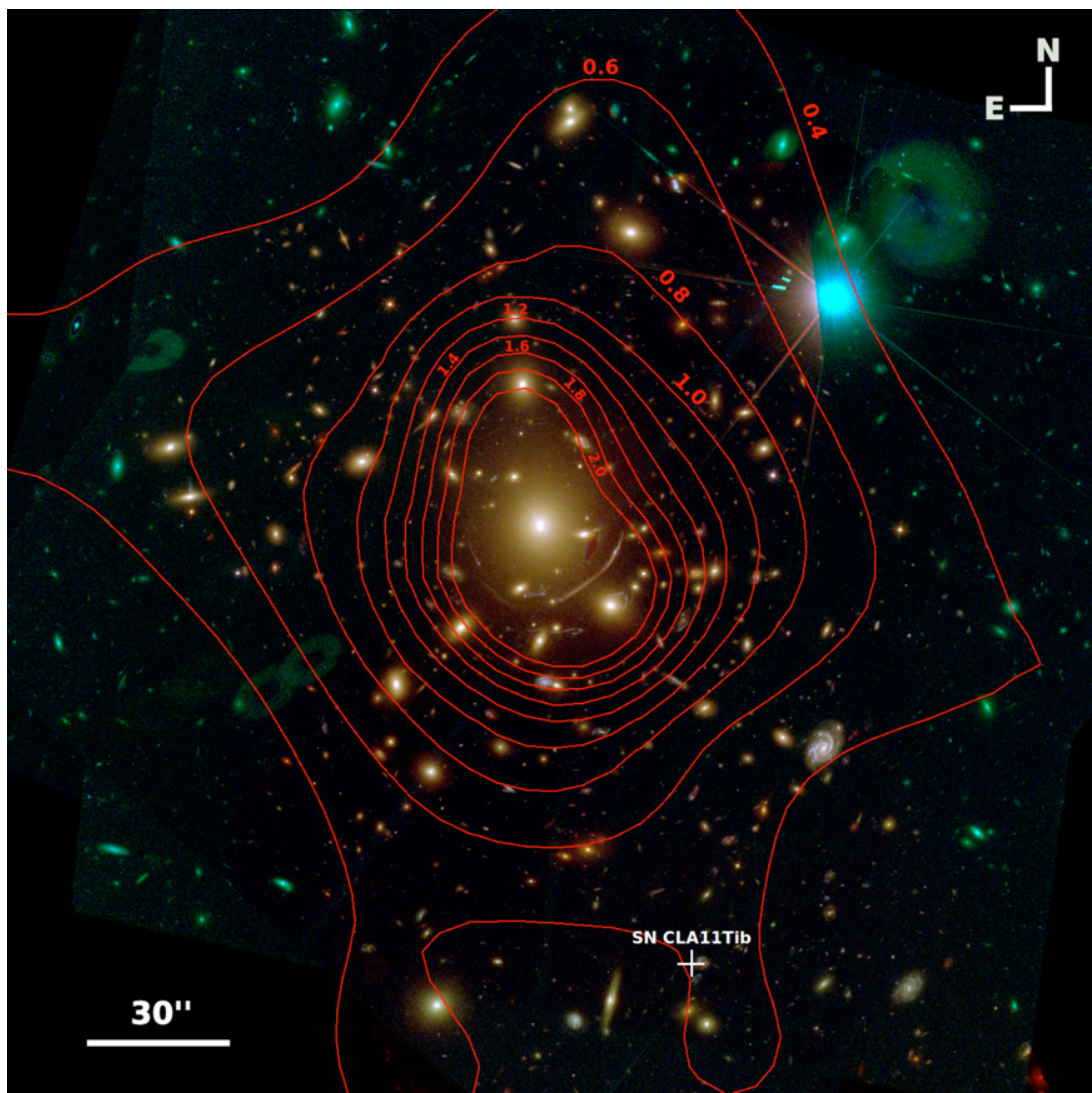

Figure 13. Magnification map for SN CLA11Tib $(z=1.143)$ behind A383, similar to Figures 10 and 12, computed from the SaWLens algorithm (Merten et al. 2009, 2011) from strong and weak-lensing constraints. The strong lens multiple images in this system were presented by Zitrin et al. (2011). (A color version of this figure is available in the online journal.)

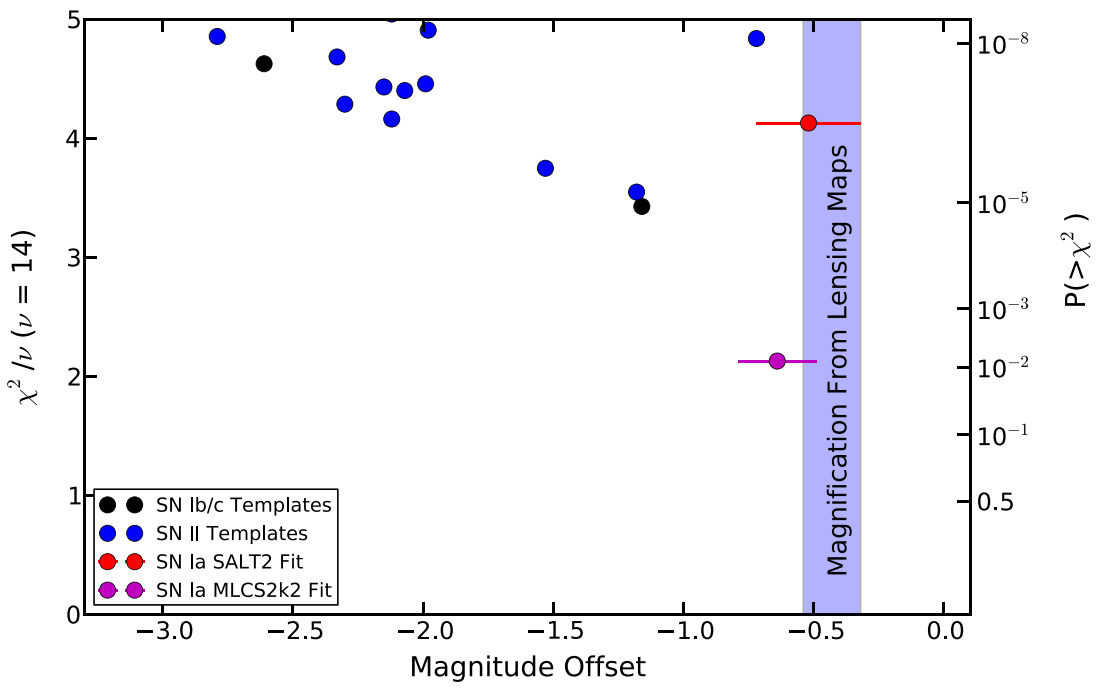

Figure 14. Reduced $\chi^{2}$ (with 14 degrees of freedom) vs. magnitude offset for SN CLA11Tib. The magnitude offset is a proxy for $\Delta m_{\mu}$ from the light-curve fit model, as described in the text. The cumulative probability for a given $\chi_{v}^{2}$ value is displayed on the right-hand side. The blue shaded region corresponds to the $1 \sigma$ range of $\Delta m_{\mu}$ derived from the lensing map in Figure 13. The black and blue points correspond to SN Ib/c and SN II models, respectively, while the red (SALT2) and magenta (MLCS2k2) points are the best-fit SN Ia models. Only the MLCS2k2 fit is consistent with both the SN light curve and the lensing map magnification prediction, but the CC SN templates do not span the full range of CC SN luminosities and light curves.

(A color version of this figure is available in the online journal.)

proposition given the large luminosity scatter of $\mathrm{CC} \mathrm{SNe} \mathrm{(Li}$ et al. 2011; Drout et al. 2011; Kiewe et al. 2012). Because of this, if we assume a CC SN fit, we cannot claim that there is any consistency or inconsistency between the lensing prediction for SN CLA11Tib and its brightness.

\section{DISCUSSION AND CONCLUSION}

SN CLO12Car clearly represents our most exciting result. We find that it is likely an SN Ia at $z=1.281$, gravitationally magnified by $\sim 1.0 \pm 0.2 \mathrm{mag}$. The magnification derived from 
two independent methods (the SN light curve and the cluster lensing maps), were consistent to within $1 \sigma$. SN CLO12Car is the first SN Ia which is both measurably magnified (i.e., with $\Delta m_{\mu}>0$ at high significance) and for which the lensing magnification can be precisely and independently derived. SN CLN12Did is also an SN Ia with a consistent (but not significant) SN-based and model-based lensing magnification, whereas the unclear classification of SN CLA11Tib does not allow for a test of the lens model prediction.

The lens models used here were constructed jointly with both strong-lensing constraints (multiple images) and weak-lensing constraints. In principle, the strong-lensing multiple images directly constrain the deflection field, which is the gradient of the potential, while the weak-lensing ellipticity measurements further out constrain the (reduced) shear, a combination of secondorder derivatives of the potential. As an alternative to the analysis presented here (where we independently tested the estimated magnifications), lensed standard candles like SNe Ia can play an important role as an additional direct, local constraint on the magnification (which is a function of the convergence $\kappa$, and the shear $\gamma$, both constructed from second-order derivatives of the potential) in the lens model (e.g., Riehm et al. 2011).

Gathering large samples of lensed SNe Ia is clearly of interest. Due to the magnification power of the foreground lenses, lensed $\mathrm{SNe}$ could be expected to be seen to higher redshifts than field $\mathrm{SNe}$ (for a given limiting magnitude), with applications to extending the redshift coverage of cosmological parameter measurements (if the lensing magnification can be estimated precisely and accurately enough, e.g., Zitrin et al. 2013) or SN rate measurements to constrain progenitor models (e.g., Graur et al. 2014; Rodney et al. 2014). There is also the exciting prospect of finding multiply imaged $\mathrm{SNe}$, with measured time delays as a new cosmographic constraint (e.g., Holz 2001; Goobar et al. 2002; Oguri \& Kawano 2003; Oguri et al. 2003).

SN Ia luminosity distances, combined with lens model magnification estimates (which depend on angular diameter distances to and between the lens and source) or time-delay distances from multiple SN images, could also open up new tests of general relativity (e.g., Daniel \& Linder 2010; Jain \& Khoury 2010; Schwab et al. 2010) or even fundamental cosmological assumptions, like the distance duality relation (e.g., Lampeitl et al. 2010; Liang et al. 2013).

Though the CLASH survey has ended, the three lensed SNe found behind CLASH clusters herald the promise of many more such discoveries in the near future, including from wide-field ground-based surveys like the Dark Energy Survey (Frieman \& Dark Energy Survey Collaboration 2013; Bernstein et al. 2012), Pan-STARRS (Rest et al. 2013 and references therein), and ultimately LSST (LSST Science Collaboration et al. 2009); ground-based surveys that target massive galaxy clusters, such as CluLeSS (S. W. Jha et al., in preparation) or SIRCLS (Graham et al. 2013); and targeted space-based surveys like the HST Frontier Fields (PI: M. Mountain; with SN followup observations in program GO-13396, PI: S. Rodney). SNe Ia were the key to the discovery of the accelerating universe; perhaps gravitationally lensed SNe Ia will play a starring role in further illuminating the dark universe, probing not just dark energy, but dark matter as well.

We would like to thank Rick Kessler for invaluable assistance with SNANA. We are grateful to Jakob Nordin for helpful discussions, and Saul Perlmutter and the Supernova Cosmology Project for communication and coordination.
This research at Rutgers University was supported through NASA/HST grant GO-12099.14 and National Science Foundation (NSF) CAREER award AST-0847157 to S.W.J. Support for S.R. and A.Z. was provided by NASA through Hubble Fellowship grants HST-HF-51312.01 and HST-HF-51334.01, respectively, awarded by the Space Telescope Science Institute, which is operated by the Association of Universities for Research in Astronomy, Inc., for NASA, under contract NAS 5-26555. L.I. thanks Basal/CATA CONICYT funding for support. This research was carried out in part at the Jet Propulsion Laboratory, California Institute of Technology, under a contract with NASA. Support for HST programs GO-12065 and GO-12099 was provided by NASA through a grant from the Space Telescope Science Institute, which is operated by the Association of Universities for Research in Astronomy, Incorporated, under NASA contract NAS5-26555. A.V.F. is also grateful for the support of NSF grant AST-1211916, the TABASGO Foundation, and the Christopher R. Redlich Fund. Support for M.N. was provided in part by grant PRIN-INAF 2010.

Some of the observations reported in this paper were obtained with the Southern African Large Telescope (SALT), through Rutgers University program 2011-3-RU-001 (PI: C. McCully). This work was supported by a NASA Keck PI Data Award, administered by the NASA Exoplanet Science Institute. Data presented herein were obtained at the W. M. Keck Observatory from telescope time allocated to the National Aeronautics and Space Administration through the agency's scientific partnership with the California Institute of Technology and the University of California. The Observatory was made possible by the generous financial support of the W. M. Keck Foundation. The authors recognize and acknowledge the very significant cultural role and reverence that the summit of Mauna Kea has always had within the indigenous Hawaiian community, and we are most privileged to have the opportunity to explore the universe from this mountain. This work is based in part on data collected at the Subaru telescope and obtained from the Subaru-Mitaka-Okayama-Kiso Archive, which is operated by the Astronomy Data Center, National Astronomical Observatory of Japan.

Additional data were obtained at the Gemini Observatory, which is operated by the Association of Universities for Research in Astronomy, Inc., under a cooperative agreement with the NSF on behalf of the Gemini partnership: the National Science Foundation (United States), the National Research Council (Canada), CONICYT (Chile), the Australian Research Council (Australia), Ministério da Ciência, Tecnologia e Inovação (Brazil), and Ministerio de Ciencia, Tecnología e Innovación Productiva (Argentina). The data were taken as part of programs GN-2012A-Q-32 and GN-2013A-Q-25.

\section{REFERENCES}

Abazajian, K. N., Adelman-McCarthy, J. K., Agüeros, M. A., et al. 2009, ApJS, 182,543

Abell, G. O., Corwin, H. G., Jr., \& Olowin, R. P. 1989, ApJS, 70, 1

Amanullah, R., Goobar, A., Clément, B., et al. 2011, ApJL, 742, L7

Balestra, I., Vanzella, E., Rosati, P., et al. 2013, A\&A, 559, L9

Bartelmann, M. 2010, CQGra, 27, 233001

Bazin, G., Palanque-Delabrouille, N., Rich, J., et al. 2009, A\&A, 499, 653

Benítez, N., Riess, A., Nugent, P., et al. 2002, ApJL, 577, L1

Bernstein, J. P., Kessler, R., Kuhlmann, S., et al. 2012, ApJ, 753, 152

Botticella, M. T., Smartt, S. J., Kennicutt, R. C., et al. 2012, A\&A, 537, A132

Cappellaro, E., Evans, R., \& Turatto, M. 1999, A\&A, 351, 459

Chornock, R., Berger, E., Rest, A., et al. 2013, ApJ, 767, 162

Conley, A., Guy, J., Sullivan, M., et al. 2011, ApJS, 192, 1

Conley, A., Sullivan, M., Hsiao, E. Y., et al. 2008, ApJ, 681, 482 
Crawford, S. M., Still, M., Schellart, P., et al. 2010, Proc. SPIE, 7737, 773725 Dahlen, T., Strolger, L.-G., Riess, A. G., et al. 2004, ApJ, 613, 189

Daniel, S. F., \& Linder, E. V. 2010, PhRvD, 82, 103523

Dilday, B., Bassett, B., Becker, A., et al. 2010, ApJ, 715, 1021

Dolphin, A. E. 2000, PASP, 112, 1383

Dressel, L. 2012, Wide Field Camera 3 Instrument Handbook for Cycle 21 v. 5.0 (Baltimore, MD: STScI)

Drout, M. R., Soderberg, A. M., Gal-Yam, A., et al. 2011, ApJ, 741, 97

Ebeling, H., Edge, A. C., Bohringer, H., et al. 1998, MNRAS, 301, 881

Ebeling, H., Edge, A. C., Mantz, A., et al. 2010, MNRAS, 407, 83

Faber, S. M., Phillips, A. C., Kibrick, R. I., et al. 2003, Proc. SPIE, 4841, 1657

Foley, R. J., \& Mandel, K. 2013, ApJ, 778, 167

Frieman, J., \& Dark Energy Survey Collaboration. 2013, in American Astronomical Society Meeting Abstracts, Vol. 221, 335.01

Gilliland, R. L., Nugent, P. E., \& Phillips, M. M. 1999, ApJ, 521, 30

Goobar, A., Mörtsell, E., Amanullah, R., \& Nugent, P. 2002, A\&A, 393, 25

Goobar, A., Paech, K., Stanishev, V., et al. 2009, A\&A, 507, 71

Graham, M. L., Pritchet, C. J., Sullivan, M., et al. 2008, AJ, 135, 1343

Graham, M. L., Sand, D. J., Pritchet, C. J., et al. 2013, ATel, 5017, 1

Graur, O., Rodney, S. A., Maoz, D., et al. 2014, ApJ, 783, 28

Guy, J., Sullivan, M., Conley, A., et al. 2010, A\&A, 523, A7

Hicken, M., Wood-Vasey, W. M., Blondin, S., et al. 2009, ApJ, 700, 1097

Holtzman, J. A., Marriner, J., Kessler, R., et al. 2008, AJ, 136, 2306

Holz, D. E. 2001, ApJL, 556, L71

Holz, D. E., \& Linder, E. V. 2005, ApJ, 631, 678

Hook, I. M., Jørgensen, I., Allington-Smith, J. R., et al. 2004, PASP, 116,425

Jain, B., \& Khoury, J. 2010, AnPhy, 325, 1479

Jha, S., Riess, A. G., \& Kirshner, R. P. 2007, ApJ, 659, 122

Jones, D. O., Rodney, S. A., Riess, A. G., et al. 2013, ApJ, 768, 166

Jönsson, J., Sullivan, M., Hook, I., et al. 2010, MNRAS, 405, 535

Jouvel, S., Host, O., Lahav, O., et al. 2014, A\&A, 562, 86

Karpenka, N. V., March, M. C., Feroz, F., \& Hobson, M. P. 2013, MNRAS, 433, 2693

Kessler, R., Bassett, B., Belov, P., et al. 2010, PASP, 122, 1415

Kessler, R., Becker, A. C., Cinabro, D., et al. 2009a, ApJS, 185, 32

Kessler, R., Bernstein, J. P., Cinabro, D., et al. 2009b, PASP, 121, 1028

Kiewe, M., Gal-Yam, A., Arcavi, I., et al. 2012, ApJ, 744, 10

Kneib, J.-P., Ellis, R. S., Santos, M. R., \& Richard, J. 2004, ApJ, 607, 697

Kolatt, T. S., \& Bartelmann, M. 1998, MNRAS, 296, 763

Kronborg, T., Hardin, D., Guy, J., et al. 2010, A\&A, 514, A44

Lampeitl, H., Nichol, R. C., Seo, H.-J., et al. 2010, MNRAS, 401, 233

Le Fèvre, O., Saisse, M., Mancini, D., et al. 2003, Proc. SPIE, 4841 1670

Li, W., Leaman, J., Chornock, R., et al. 2011, MNRAS, 412, 1441

Liang, N., Li, Z., Wu, P., et al. 2013, MNRAS, 436, 1017
LSST Science Collaboration, Abell, P. A., Allison, J., et al. 2009 , arXiv:0912.0201

Mannucci, F. 2005, in ASP Conf. Ser. 342, 1604-2004: Supernovae as Cosmological Lighthouses, ed. M. Turatto, S. Benetti, L. Zampieri, \& W. Shea (San Francisco, CA: ASP), 140

Martel, H., \& Premadi, P. 2008, ApJ, 673, 657

Medezinski, E., Umetsu, K., Nonino, M., et al. 2013, ApJ, 777, 43

Meneghetti, M., Rasia, E., Merten, J., et al. 2010, A\&A, 514, A93

Merten, J., Cacciato, M., Meneghetti, M., Mignone, C., \& Bartelmann, M. 2009, A\&A, 500, 681

Merten, J., Coe, D., Dupke, R., et al. 2011, MNRAS, 417, 333

Nordsieck, K. 2012, in AIP Conf. Proc. 1429, Stellar Polarimetry: From Birth to Death, ed. J. L. Hoffman, J. Bjorkman, \& B. Whitney (Melville, NY: AIP), 248

Oguri, M., \& Kawano, Y. 2003, MNRAS, 338, L25

Oguri, M., \& Marshall, P. J. 2010, MNRAS, 405, 2579

Oguri, M., Suto, Y., \& Turner, E. L. 2003, ApJ, 583, 584

Östman, L., Nordin, J., Goobar, A., et al. 2011, A\&A, 526, A28

Perlmutter, S., Aldering, G., Goldhaber, G., et al. 1999, ApJ, 517, 565

Pogge, R. W., Atwood, B., Brewer, D. F., et al. 2010, Proc. SPIE, 7735, 77350

Postman, M., Coe, D., Benítez, N., et al. 2012, ApJS, 199, 25

Quimby, R. M., Werner, M. C., Oguri, M., et al. 2013, ApJL, 768, L20

Refsdal, S. 1964, MNRAS, 128, 307

Rest, A., Scolnic, D., Foley, R. J., et al. 2013, arXiv:1310.3828

Riehm, T., Mörtsell, E., Goobar, A., et al. 2011, A\&A, 536, A94

Riess, A. G., Filippenko, A. V., Challis, P., et al. 1998, AJ, 116, 1009

Riess, A. G., Nugent, P. E., Gilliland, R. L., et al. 2001, ApJ, 560, 49

Riess, A. G., Strolger, L.-G., Casertano, S., et al. 2007, ApJ, 659, 98

Rodney, S. A., Riess, A. G., Dahlen, T., et al. 2012, ApJ, 746, 5

Rodney, S. A., Riess, A. G., Strolger, L.-G., et al. 2014, ApJ, submitted (arXiv:1401.7978)

Rubin, D., Knop, R. A., Rykoff, E., et al. 2013, ApJ, 763, 35

Sako, M., Bassett, B., Becker, A., et al. 2008, AJ, 135, 348

Sako, M., Bassett, B., Becker, A. C., et al. 2014, arXiv:1401.3317

Sako, M., Bassett, B., Connolly, B., et al. 2011, ApJ, 738, 162

Schwab, J., Bolton, A. S., \& Rappaport, S. A. 2010, ApJ, 708, 750

Smith, M., Bacon, D. J., Nichol, R. C., et al. 2014, ApJ, 780, 24

Stanishev, V., Goobar, A., Paech, K., et al. 2009, A\&A, 507, 61

Sullivan, M., Ellis, R., Nugent, P., Smail, I., \& Madau, P. 2000, MNRAS, 319, 549

Sullivan, M., Guy, J., Conley, A., et al. 2011, ApJ, 737, 102

Suzuki, N., Rubin, D., Lidman, C., et al. 2012, ApJ, 746, 85

Umetsu, K., Medezinski, E., Nonino, M., et al. 2012, ApJ, 755, 56

Wood-Vasey, W. M., Miknaitis, G., Stubbs, C. W., et al. 2007, ApJ, 666, 694

Zitrin, A., Broadhurst, T., Coe, D., et al. 2011, ApJ, 742, 117

Zitrin, A., Broadhurst, T., Umetsu, K., et al. 2009, MNRAS, 396, 1985

Zitrin, A., Redlich, M., \& Broadhurst, T. 2013, arXiv:1311.5224 